\title{
Anticancer Potential of Lipophilic Constituents of Eleven Shellfish Species Commonly Consumed in Korea
}

\author{
Juhyun Shin ${ }^{1}{ }^{\circledR}$, Min-Ho Song ${ }^{2}$, Ji-Woo Yu ${ }^{2}$, Eun-Young Ko ${ }^{3}{ }^{\circledR}$, Xiaomin Shang ${ }^{4}$, Jae-Wook Oh ${ }^{1}{ }^{1}$, \\ Young-Soo Keum ${ }^{2}$ and Ramesh Kumar Saini ${ }^{2, *(D)}$ \\ 1 Department of Stem Cell and Regenerative Biotechnology, Konkuk University, Seoul 143-701, Korea; \\ junejhs@konkuk.ac.kr (J.S.); ohjw@konkuk.ac.kr (J.-W.O.) \\ 2 Department of Crop Science, Konkuk University, Seoul 143-701, Korea; hlhkkl@konkuk.ac.kr (M.-H.S.); \\ wooody96@konkuk.ac.kr (J.-W.Y.); rational@konkuk.ac.kr (Y.-S.K.) \\ 3 Department of Food Science and Biotechnology of Animal Resources, Konkuk University, Seoul 143-701, \\ Korea; key523@konkuk.ac.kr \\ 4 Jilin Provincial Key Laboratory of Nutrition and Functional Food, Jilin University, Changchun 130062, China; \\ xmshang@jlu.edu.cn \\ * Correspondence: saini1997@konkuk.ac.kr
}

Citation: Shin, J.; Song, M.-H.; Yu, J.-W.; Ko, E.-Y.; Shang, X.; Oh, J.-W.; Keum, Y.-S.; Saini, R.K. Anticancer Potential of Lipophilic Constituents of Eleven Shellfish Species Commonly Consumed in Korea. Antioxidants 2021, 10, 1629. https:// doi.org/10.3390/antiox10101629

Academic Editor: Evangelos Zoidis

Received: 24 September 2021

Accepted: 13 October 2021

Published: 15 October 2021

Publisher's Note: MDPI stays neutral with regard to jurisdictional claims in published maps and institutional affiliations.

Copyright: (c) 2021 by the authors. Licensee MDPI, Basel, Switzerland. This article is an open access article distributed under the terms and conditions of the Creative Commons Attribution (CC BY) license (https:/ / creativecommons.org/licenses/by/ $4.0 /)$.

\begin{abstract}
The present study was aimed to investigate the composition and contents and the major lipophilic compounds, including the sterols, fatty acids, and tocols of shellfish species. Moreover, to explore the antitumor activity of these lipophilic constituents, their cytotoxicity potentials were determined against five different human cancer cells, including colon carcinoma (HCT116), epithelial melanoma (A2058), glioblastoma multiforme (T98G), lung carcinoma (A549), and adenocarcinoma (HeLa). The results show a significant variation in the contents and composition of lipophilic constituents among the studied species. The highest omega-3 (n-3) polyunsaturated fatty acids (PUFAs) were recorded from arrow squid and pacific oysters, accounting for $53.2 \%$ and $53.0 \%$ of their total fatty acids, respectively. However, the highest cholesterol content was also recorded in arrow squid (154.4 mg/100 g; 92.6\% of total sterols). In contrast, in the Japanese littleneck, Yesso scallop, and common orient clam, cholesterol was just $17.1 \%, 18.3 \%$, and $18.9 \%$ of total sterols, respectively, making them the richest source of non-cholesterol sterols (NCS). Lipids extracted from shellfish species showed $\mathrm{ABTS}^{+} \bullet^{\bullet}$ and $\mathrm{DPPH}^{\bullet}$-scavenging activities. In the cytotoxicity analysis, lipids extracted from the Argentine red shrimp showed the highest cytotoxicity against glioblastoma multiforme T98G cells, with an $\mathrm{IC}_{50}$ value of $12.3 \mu \mathrm{g} / \mathrm{mL}$. The composition and cytotoxicity data reported herein may help explore the nutritional and anticancer potentials of shellfish species.
\end{abstract}

Keywords: cholesterol; phytosterols; omega-3 fatty acids; tocopherol; mollusks; crustaceans; DPPH ${ }^{\bullet}$ scavenging activities; $\mathrm{ABTS}^{+} \bullet$ scavenging activities; glioblastoma multiforme (T98G); lung carcinoma (A549)

\section{Introduction}

Shellfish (mollusks and crustaceans) species are the key dietary source of omega-3 (n-3) very-long-chain (VLC)-polyunsaturated fatty acids (PUFAs), mainly eicosapentaenoic (EPA; C20:5n3), and docosahexaenoic acids (DHA; C22:6n3), which play a crucial role in reducing the risk of cancer and cardiovascular diseases (CVD). Moreover, DHA-derived specialized pro-resolving mediators (SPMs, e.g., protectins, resolvins, and maresins) are critically crucial for neonatal brain development and mental and cognitive development [1] and neuroprotection [2,3].

Though with the richness of VLC-n-3 PUFAs, shellfish are considered a vital component of a healthy diet, the high cholesterol content of some species is generally cited as a reason to limit their intake. However, in addition to cholesterol, shellfish species contain a significant amount of other sterols (called non-cholesterols sterols; NCS); some 
are unique to marine species [4], derived from the food they consume (e.g., microalgae) and from endogenous metabolism. Plant-derived sterols (called phytosterols) are well known to reduce low-density plasma lipoprotein-cholesterol (LDL-C) levels [5-7] and thereby lower the risk of CVD. Moreover, as antioxidants, phytosterols are well-known to scavenge harmful reactive oxygen species (ROS) [8]. Furthermore, animal and human studies have demonstrated the anticancer [9] and anti-inflammatory [10] effects of phytosterols. Considering these facts, consuming a phytosterol-rich diet may provide health benefits.

Tocopherols and tocotrienols (collectively known as tocols, tocochromanols, or vitamin E) are key components of plant- and animal-derived lipids, which scavenge ROS, thus protecting lipids from oxidative degradation. Given their functional role in controlling cellular oxidative stress, a diet rich in tocols minimizes the incidence of cancer, CVD, and neurodegenerative diseases [11-13].

To the best of our knowledge, only a few detailed studies are available on the sterol composition of shellfish species [4], while most studies reported only cholesterol and few other sterols, such as sitosterol, campesterol, or stigmasterol [14-16], while many shellfish species contain still other sterols at significant levels. Moreover, culturing conditions and dietary factors can affect the sterol content of shellfish species harvested from different natural geographical locations [4]. Similarly, the detailed composition of many shellfish species commonly consumed in Korea is not available. Furthermore, similarly to sterols, fatty acids' composition in shellfish may vary with season [17], sex (e.g., male vs. female crabs) [18], culturing conditions (e.g., wild vs. cultured, and diet quality) $[19,20]$, and several other factors [21].

Considering the above, the present investigation aims to analyze the composition and contents of the major lipophilic compounds, including sterols, fatty acids, and tocols, of shellfish species, commonly consumed in Korea. Sterols were analyzed by gas chromatography (GC)-mass spectrometry (MS), fatty acids by GC-flame ionization detection (FID) and GC-MS, and tocols by high-performance liquid chromatography (HPLC)-diodearray detection (DAD). Moreover, to explore the antitumoral activities of the lipophilic constituents of shellfish species, the cytotoxicity potentials of the extracted lipids were determined against five different human cancer cells, including colon carcinoma (HCT116), epithelial melanoma (A2058), glioblastoma multiforme (T98G), lung carcinoma (A549), and adenocarcinoma (HeLa). The fatty acids, sterols, and tocols composition, and the cytotoxicity data reported herein may help explore shellfish species' nutritional and anticancer potentials.

\section{Materials and Methods}

\subsection{Reagents, Standards, and Raw Material}

Authentic standards of sterols including cholesterol (>99\% purity), cholestanol ( $>99 \%$ purity), brassicasterol (>98\% purity), ergosterol (quality level of MQ300), and campesterol ( $90 \%$ purity) and fatty acids (37-Component FAME Mix, CRM47885, quality level of MQ100) were obtained from Merck Ltd., Seoul, South Korea. A mixed-tocols solution containing $\delta$-, $\gamma-, \beta-$, and $\alpha$-tocotrienol and $\delta-, \gamma-, \beta$-, and $\alpha$-tocopherol (purity adjusted) was purchased from ChromaDex, Inc. (Irvine, CA, USA). All organic solvents used for the extractions were HPLC grade and obtained from Daejung Chemicals and Metals Co., Ltd., Siheung-si, Korea.

A total of eleven shellfish species consumed in Korea were procured from the Garak market, Songpa-gu, Seoul, in October 2019 (Table 1). Two kilograms of each species were brought to the lab, the edible flesh was manually separated and homogenized using a food processor, and $30-\mathrm{g}$ portion was precisely aliquoted to falcon tubes and stored at $-80^{\circ} \mathrm{C}$ until analysis. 
Table 1. List of shellfish species used in the present investigation.

\begin{tabular}{ccc}
\hline Sample No. & Common Name & Zoological Name \\
\hline S1 & far eastern mussel & Mytilus coruscus (Gould, 1861) \\
S2 & horned turban (topshell) & Turbo cornutus (Lightfoot, 1786) \\
S3 & Japanese littleneck & Venerupis philippinarum (Adams \& Reeve, 1850) \\
S4 & Yesso scallop & Mizuhopecten yessoensis (Jay, 1857) \\
S5 & common orient clam & Meretrix lusoria (Roeding 1798) \\
S6 & Gazami crab & Portunus trituberculatus (Miers, 1876) \\
S7 & long-arm octopus & Octopus minor (Sasaki, 1920) \\
S8 & abalone & Haliotis discus, Linnaeus, 1758 \\
S9 & arrow squid & Heterololigo bleekeri (Keferstein, 1866) \\
S10 & Pacific oyster & Magallana gigas (Thunberg, 1793) \\
S11 & Argentine red shrimp & Pleoticus muelleri (Bate, 1888) \\
\hline
\end{tabular}

\subsection{Extraction of Crude Lipids (Lipophilic Compounds)}

Crude lipids, containing lipophilic compounds, were extracted from the edible portions of the studied shellfish species following our optimized protocol [22], with minor modifications; it was initially based on a previous report [23]. The detailed extraction procedure is illustrated in Appendix A. The butylated hydroxytoluene (BHT: $w / v$; synthetic antioxidant) was added to the extraction solvent to minimize the degradation of lipophilic compounds [24]. The crude lipids utilized for the cell culture analysis were extracted without using the BHT. The extracted crude lipids were utilized to analyze their fatty acids, tocols, and sterols, and to determine their anticancer potentials, as shown in Appendix A. Tocols were analyzed by HPLC without hydrolysis, as it can degrade the tocols [23]. The crude lipids were converted to fatty acid methyl esters (FAMEs) using a commercially available boron trifluoride-methanol solution (14\% in methanol; Merck Ltd., Seoul, South Korea) as per the manufacturer's guidelines, with minor modifications (Appendix B). Similarly, before the GC-MS analysis, the crude lipids were hydrolyzed for sterol analysis, as mentioned in Appendix B [23].

\subsection{HPLC Analysis of Tocols}

The chromatographic separation of tocols was achieved utilizing an HPLC system (Model 1100; Agilent Technologies Canada, Inc., Mississauga, ON, Canada) equipped with a YMC C 30 column $(250 \times 4.6 \mathrm{~mm}, 5 \mu \mathrm{m}$; YMC, Wilmington, NC, USA) and a DAD. The solvent system, gradient elution pattern, flow rate, and detection wavelengths were used as previously optimized [25].

\subsection{FAMEs Determinations by Gas Chromatography (GC)-Flame Ionization Detection (FID) and GC-Mass Spectrometry (MS)}

FAMEs were quantitatively analyzed by GC (Agilent 7890B, Agilent Technologies, Santa Clara, CA, USA) equipped with an FID and an SP-2560 capillary column (100 m, $0.20 \mu \mathrm{m}$ film thickness, $0.25 \mathrm{~mm}$ ID; Merck KGaA, Darmstadt, Germany). The thermal program and other FID parameters were used as optimized in recent investigations [26]. For precise identification, compounds' mass spectra were recorded through the GS-MS system (QP2010 SE; Shimadzu, Japan), following a thermal program of GC-FID analysis. The identities of FAMEs were confirmed by comparing their fragmentation pattern with the authentic standards.

\subsection{Calculation of Fat Quality Indices}

Fat-quality indices, including total polyunsaturated fatty acids (PUFAs)/total saturated fatty acids (SFAs), n-3 PUFAs/n-6 PUFAs, hypocholesterolemic (h)/ hypercholesterolemic $(\mathrm{H})$ fatty acids ratios, atherogenic index $(\mathrm{AI})$, and thrombogenic index (TI), were calculated from the fatty acid-profiling results [27-30]. The $\mathrm{h} / \mathrm{H}, \mathrm{AI}$, and TI were calculated as per the following equations:

$$
\mathrm{h} / \mathrm{H}=\frac{\mathrm{C} 18: 1+\sum \text { PUFA }}{\mathrm{C} 14: 0+\mathrm{C} 16: 0}
$$




$$
\begin{gathered}
\mathrm{AI}=\frac{(4 \times \mathrm{C} 14: 0)+\mathrm{C} 16: 0}{\sum \text { MUFAs }+\sum \text { PUFAs }} \\
\mathrm{TI}=\frac{\mathrm{C} 14: 0+\mathrm{C} 16: 0+\mathrm{C} 18: 0}{\left(0.5 \times \sum \text { MUFAs }\right)+\left(0.5 \times \sum \mathrm{n}-6 \text { PUFAs }\right)+\left(3 \times \sum \mathrm{n}-3 \text { PUFAs }\right)+\left(\frac{\sum \mathrm{n}-3 \text { PUFAs }}{\sum \mathrm{n}-6 \text { PUFAs }}\right)}
\end{gathered}
$$

\subsection{GC-MS Analysis of Sterols}

Sterols were analyzed after silylation, utilizing a QP2010 SE GC-MS equipped with a fused silica Rxi-5ms column (30 m, 0.5- $\mu \mathrm{m}$ film thickness, 0.25-mm ID; Restek Corporation, Bellefonte, PA, USA). Helium was used as a carrier gas, maintained at the pressure control flow of $33.5 \mathrm{~cm} / \mathrm{min}(7.8-\mathrm{mL} / \mathrm{min}$ total flow). The injector and MS ion source were precisely maintained at 260 , while the MS interface was maintained at $280{ }^{\circ} \mathrm{C}$. The column oven temperature was kept at $120^{\circ} \mathrm{C}$ for $1 \mathrm{~min}$, then progressively increased to $300^{\circ} \mathrm{C}$ with a linear increase of $15^{\circ} \mathrm{C} / \mathrm{min}$, and held at $300^{\circ} \mathrm{C}$ for $27 \mathrm{~min}$ [31]. One $\mu \mathrm{L}$ of samples and standards were injected in a 1:5 split ratio. Their fragmentation pattern was compared with authentic standards and reference databases (NIST08, NIST08S, and Wiley9).

\subsection{Total Equivalent Antioxidant Capacities (TEAC)}

The total equivalent antioxidant capacities (TEAC) of the lipids extracted from the shellfish species was determined using 1,1-diphenyl-2-picrylhydrazyl- ( $\left.\mathrm{DPPH}^{\bullet}\right)$ and 2,2' azino-bis(3-ethylbenzothiazoline-6-sulphonic acid)- (ABTS ${ }^{\bullet+}$ ) scavenging assays following our previously optimized procedures [32], originally based on the studies of Thaipong et al. [33] with minor modifications.

Briefly, for the $\mathrm{ABTS}^{+} \bullet$ decolorization assay, $1950 \mu \mathrm{L}$ of freshly prepared $\mathrm{ABTS}^{+\bullet}$ solution was allowed to react with $50 \mu \mathrm{L}(1.5 \mathrm{mg})$ of extracted lipids for $2 \mathrm{~h}$ in the dark. Later, the absorbance was measured at $734 \mathrm{~nm}$, using a spectrophotometer (UV-2550, Shimadzu, Japan). A linear standard calibration curve was prepared using Trolox as standard in the range of $0.01-10 \mu \mathrm{g} / \mathrm{mL}$. The results are expressed as the mg-of-Trolox equivalents (TE)/g of the lipids.

For the $\mathrm{DPPH}^{\bullet}$-scavenging activities, $1950 \mu \mathrm{L}$ of freshly prepared $\mathrm{DPPH}^{\bullet}$ solution $(0.1 \mathrm{mM})$ was allowed to react with $50 \mu \mathrm{L}(1.5 \mathrm{mg})$ of extracted lipids for $80 \mathrm{~min}$ in the dark. Later, the absorbance was measured at $517 \mathrm{~nm}$ using the spectrophotometer. A linear standard calibration curve was prepared using Trolox as standard in the range of $0.01-10 \mu \mathrm{g} / \mathrm{mL}$. The results are expressed as the mg-of-Trolox equivalents (TE)/g of the lipids.

\subsection{Cytotoxicity Studies}

The HCT116 (Colorectal carcinoma), A2058 (Melanoma), A549 (lung carcinoma), T98G (Glioblastoma multiforme), and Hela (Adenocarcinoma) cell lines were purchased from the American Type Culture Collection (ATCC, Manassas, VI, USA). Cells were cultured in McCoy's 5A Medium, Dulbecco's Modified Eagle's Medium, or Minimum Essential Medium Eagle, supplemented with $10 \%$ FBS, $1 \mathrm{mM}$ sodium pyruvate, and 100 units $/ \mathrm{mL}$ penicillin/streptomycin, accordingly, and incubated in a $5 \%-\mathrm{CO}_{2}$ incubator at $37{ }^{\circ} \mathrm{C}$. Prior to treatment, cells were seeded at $3 \times 10^{3}$ cells/per well density in 96-well plate and incubated overnight. Treatments were performed by carefully washing the cells with Dubelcco's Phosphate Buffered Saline twice and adding lipids in media without FBS to the washed wells. Lipids were dissolved at a concentration of $20 \mathrm{mg} / \mathrm{mL}$ in DMSO, and cells were treated at concentrations ranging from 10 to $100 \mu \mathrm{g} / \mathrm{mL}$ in $0.5 \%$ DMSO for $24 \mathrm{~h}$. Cytotoxicity was assayed by adding $10 \mu \mathrm{L}$ of WST-1 EZ-cytox (DoGen, Suwon, Korea) per well with $90 \mu \mathrm{L}$ of media without FBS and incubated at $37^{\circ} \mathrm{C}$ for $40 \mathrm{~min}$. The absorbance at $450 \mathrm{~nm}$ and reference wavelength at $600 \mathrm{~nm}$ were measured using a Microplate Spectrophotometer (Biotek, VT, USA). Experiments were performed in three technical repeats for more than three biological repeats. The most representative biological repeats were used to calculated $\mathrm{IC}_{50}$ concentration by mean of the AAT-Bioquest ${ }^{\circledR}$ online tool. 


\subsection{Statistical Analysis and Quality Control}

A total of three replicate extraction and analysis were performed for each shellfish species. The results were analyzed using IBM SPSS statistics (version 25; IBM Corp., Armonk, NY, USA) employing a one-way analysis of variance (ANOVA), considering a significance level of 0.05 (Turkey HSD).

The use of the GC-MS method for quantifying sterols was recently validated in terms of accuracy, linearity, precision, and stability [26]. The recovery of sterols was precisely monitored and normalized using $5 \beta$-cholestan- $3 \alpha$-ol as an internal standard.

\section{Results and Discussion}

\subsection{Qualitative Confirmation of Sterols by GC-MS}

In this study, 11 shellfish species were analyzed for their compositions of sterols utilizing GC-MS after derivatization with trimethylsiloxy groups [TMS; - $\mathrm{O}-\mathrm{Si}\left(\mathrm{CH}_{3}\right)_{3}$ ]. In the mass spectrometric identification of sterols, the loss of trimethylsilanol (TMS-OH) is a common feature, resulting in dominant ion fragments at $m / z$ [M-90 $]^{\bullet+}[34]$. The $\Delta 5$-steryl (TMS derivatives) give a characteristic fragmentation involving the loss of the $\mathrm{C}-1, \mathrm{C}-2$, and C-3 the sterol. The A-ring with the TMS-group yielded intense ions at $m / z 129$ for the TMS derivative-containing fragment and $m / z[\mathrm{M}-129]^{\bullet+}$ for the remaining portion of the sterol compound (Figures 1 and 2) [34].

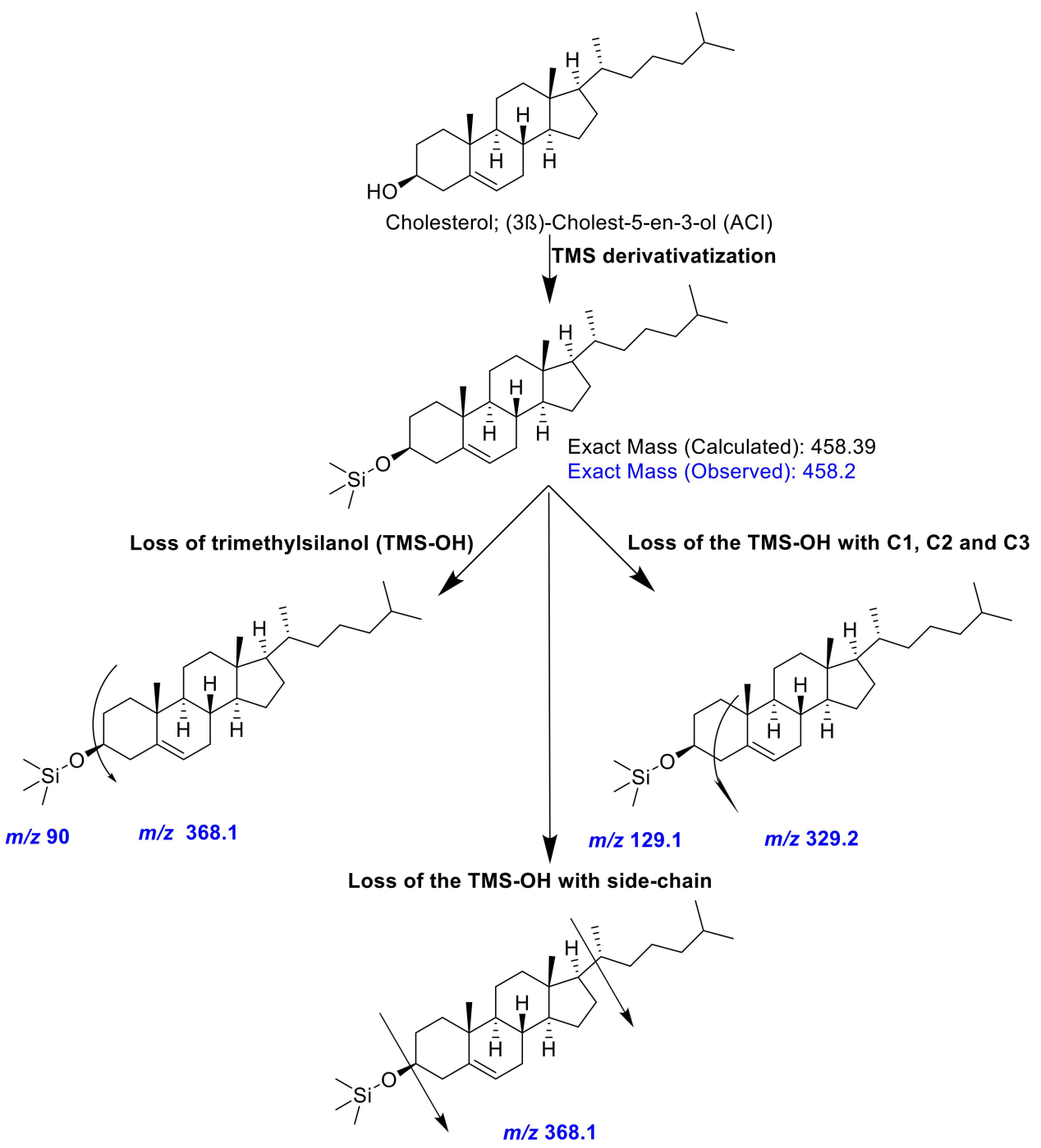

Figure 1. A representative mass-fragmentation pattern of cholesterol (trimethylsiloxy [TMS] derivative) observed in the present investigation. The colored text explains the observed $m / z$ values. 

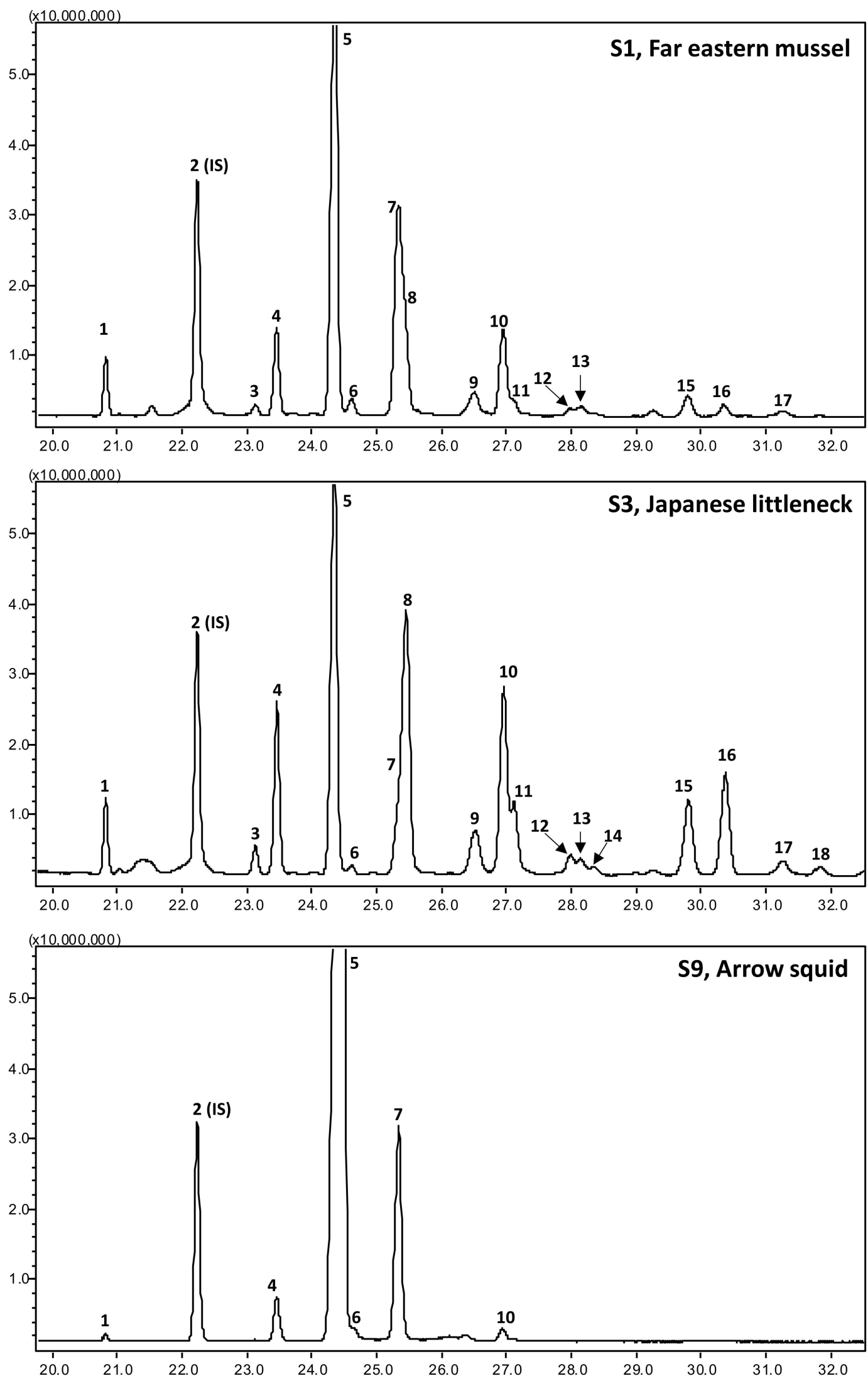

Figure 2. The representative gas chromatography (GC), mass spectrometry (MS), and total ion chromatograms (TIC) of the sterols of the far eastern mussel, Japanese littleneck, and arrow squid. The peak numbers (1 to 18) correspond to Table 2. IS: internal standard. 
Table 2. List of identified and quantified sterols from the studied species.

\begin{tabular}{|c|c|c|c|c|c|c|}
\hline Peak No. & Sterol & CAS Registry Number ${ }^{c}$ & Another Name & Calculated Exact Mass of Sterol-TMS ${ }^{d}$ & Observed Exact Mass & RT (min) \\
\hline 1 & norcholestadienol $^{\text {a }}$ & $38788-81-7$ & (22E)-24-Norcholesta-5,22-dien-3ß-ol & 442.34 & 442.1 & 20.81 \\
\hline 3 & occelasterol $^{\mathrm{a}}$ & $54278-89-6$ & (22Z)-27-Norergosta-5,22-dien-3ß-ol & 456.38 & 456.1 & 23.11 \\
\hline 4 & 22-dehydrocholesterol ${ }^{\text {a }}$ & $34347-28-9$ & Cholesta-5,22E-dien-3 $\beta$-ol & 456.38 & 456.1 & 23.44 \\
\hline 5 & cholesterol $^{\mathrm{b}}$ & $57-88-5$ & Cholest-5-en-3-ol & 458.39 & 458.2 & 24.37 \\
\hline 6 & cholestanol $^{\mathrm{b}}$ & $80-97-7$ & $(5 \alpha)$-cholestan-3ß-ol (Dihydrocholesterol) & 460.41 & 460.2 & 24.60 \\
\hline 7 & desmosterol $^{\text {a }}$ & $313-04-2$ & Cholesta-5,24-dien-3ß-ol & 456.38 & 456.1 & 25.32 \\
\hline 8 & brassicasterol $^{\mathrm{b}}$ & $474-67-9$ & Ergosta-5,22E-dien-3ß-ol & 470.39 & 470.2 & 25.41 \\
\hline 9 & ergosterol $^{\mathrm{b}}$ & $57-87-4$ & (22E)-ergosta-5,7,22-trien-3ß-ol & 468.38 & 468.1 & 26.49 \\
\hline 10 & 24-methylene-cholesterol a & $474-63-5$ & 24-Methylene-cholest-5-en-3ß-ol & 470.39 & 470.2 & 26.92 \\
\hline 12 & poriferasterol $^{\mathrm{a}}$ & $481-16-3$ & (3ß,22E,24R)-Stigmasta-5,22-dien-3-ol & 484.41 & 484.2 & 27.96 \\
\hline 13 & unidentified 1 & - & - & - & 468.1 & 28.12 \\
\hline 14 & unidentified 2 & - & - & - & 470.1 & 28.32 \\
\hline 15 & clionasterol $^{\mathrm{a}}$ & $83-47-6$ & (24S)-Stigmast-5-en-3 $\beta$-ol ( $\gamma$-Sitosterol) & 486.43 & 486.2 & 29.77 \\
\hline 16 & isofucosterol $^{\mathrm{a}}$ & 481-14-1 & (24Z)-Ethylidene-cholest-5-en-3b-ol & 484.04 & 484.1 & 30.33 \\
\hline 17 & unidentified 3 & - & - & - & 484.1 & 31.22 \\
\hline 18 & unidentified 4 & - & - & - & 482.1 & 31.81 \\
\hline
\end{tabular}

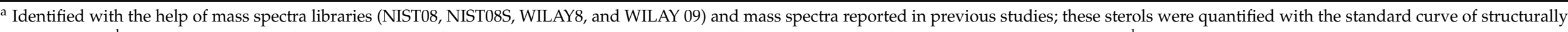

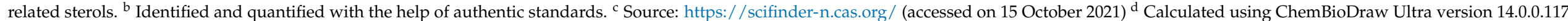
(Perkin Elmer Inc., Waltham, MA, USA). TMS: derivatization with trimethylsiloxy groups [TMS; $\left.-\mathrm{O}-\mathrm{Si}\left(\mathrm{CH}_{3}\right)_{3}\right]$. RT: retention time. 
In the present study, a total of 18 sterol compounds were detected among the studied shellfish species (Table 2). Among these sterols, 14 compounds were identified with the help of authentic standards, and NIST08, NIST08S, WILAY8, and WILAY 09 mass spectra library. Moreover, the mass spectral and gas chromatographic patterns reported in previous studies were found valuable in identifying the sterols [4,35]. Especially, stigmasterol and poriferasterol have nearly similar retention time and mass spectrum profiles, as do sitosterol and clionasterol [4], making it difficult to confirm the identity by GC-MS. Hence, peak assignments for these sterols were based on the previous reports of the isomers occurring in shellfish species [4,35].

The representative total ion chromatograms (TIC) and mass spectrum of major sterols identified in this study are given in Figures 2 and 3, respectively. In addition, the mass spectrum of four minor unidentified sterols are given in Appendix C. In the present study, mass spectrum and elution order were validated for all identifications; however, the possibilities of sterol compound co-elution and isomeric variation suggest that the presented sterol identities should be considered tentative. Further analysis with the support of NMR could provide further information for confirming their identities.

\subsection{Sterol Contents in Studied Mollusk and Crustacea}

In the present study, the studied shellfish species showed significant variation in their sterol compositions and contents. Cholesterol was the most dominant sterol in most of the studied shellfish species (Table 3). The highest cholesterol content was recorded in arrow squid (154.4 mg/100 g) and Argentine red shrimp (110.3 mg/100 g), which accounted for $92.6 \%$ and $90.3 \%$ of total sterols, respectively. In contrast, in the Japanese littleneck, Yesso scallop, and common orient clam, cholesterol was just $17.1 \%, 18.3 \%$, and $18.9 \%$ of total sterols, respectively, which makes them the richest source of NCS. The brassicasterol, 24-methylene-cholesterol, 22-dehydrocholesterol, and isofucosterol were the dominant NCS among these species. In addition, a significant amount of desmosterol was recorded in the far eastern mussel $(19.4 \mathrm{mg} / 100 \mathrm{~g})$, arrow squid $(16.8 \mathrm{mg} / 100 \mathrm{~g})$, and horned turban (14.7 mg/100 g).

Only a few detailed studies are available on the sterol compositions of shellfish species [4], while most studies reported cholesterol and few other sterols [14-16]. The cholesterol contents recorded in the present investigation are in agreement with previous studies on the Japanese littleneck and Yesso scallop [14]. Philips et al. [4] recorded the sterol composition of shellfish species for the United States Department of Agriculture (USDA) National Nutrient Database. In this USDA study, the high content of cholesterol (129 mg/100 g FW) was recorded in shrimp, whose total sterol content was $134 \mathrm{mg} / 100 \mathrm{~g}$ FW. In agreement therewith, we also recorded a significant amount of cholesterol (110.3 mg/100 g FW) in the Argentine red shrimp, whose total sterol content was $122.2 \mathrm{mg} / 100 \mathrm{~g}$ FW. Similarly, Bragagnolo and Rodriguez-Amaya [16] recorded a substantial amount of cholesterol (114-134 mg/100 g) in several species of wild shrimp.

The high contents of cholesterol is often cited as a reason to limit the consumption of shellfish species, as cholesterol is generally considered a risk factor for developing CVD [36], and known to initiate pathophysiological angiogenesis [37]. The American Heart Association (AHA) dietary guidelines (2000 edition) advised the consumption of $<300 \mathrm{mg} /$ day of cholesterol to minimize elevations in blood cholesterol [38]. Interestingly, in the 2015 edition of dietary guidelines, these restrictions were not included [39]. Interestingly, even if the recommendation of the consumption of $<300 \mathrm{mg} /$ day of cholesterol is considered, a serving size of $85 \mathrm{~g}$ of far eastern mussel, Japanese little neck, Yesso scallop, common orient clam, or pacific oyster would contribute $\approx 10 \%$ of the daily maximum, while the remaining species would contribute $\approx 19 \%$ (horned turban, Gazmi crab, and long arm octopus), 31\% (Argentine red shrimp), and 43\% (arrow squid) of daily value. 


\subsection{Fatty Acids}

In the present investigation, 18 fatty acids were identified from the edible flesh of shellfish species (Table 4, Figure 4). Among the studied species, DHA, EPA, and palmitic acid (C16:0) were most dominant. Together, these three fatty acids accounted for $30.7 \%$ (abalone) to $81.7 \%$ (arrow squid) of the total fatty acids. Amid the highest proportions of palmitic acid (29.3\%), the total SFAs were recorded highest (37.6) in the lipids extracted from arrow squid. Interestingly, the highest amount of DHA (39.6\%) was also recorded from arrow squid.
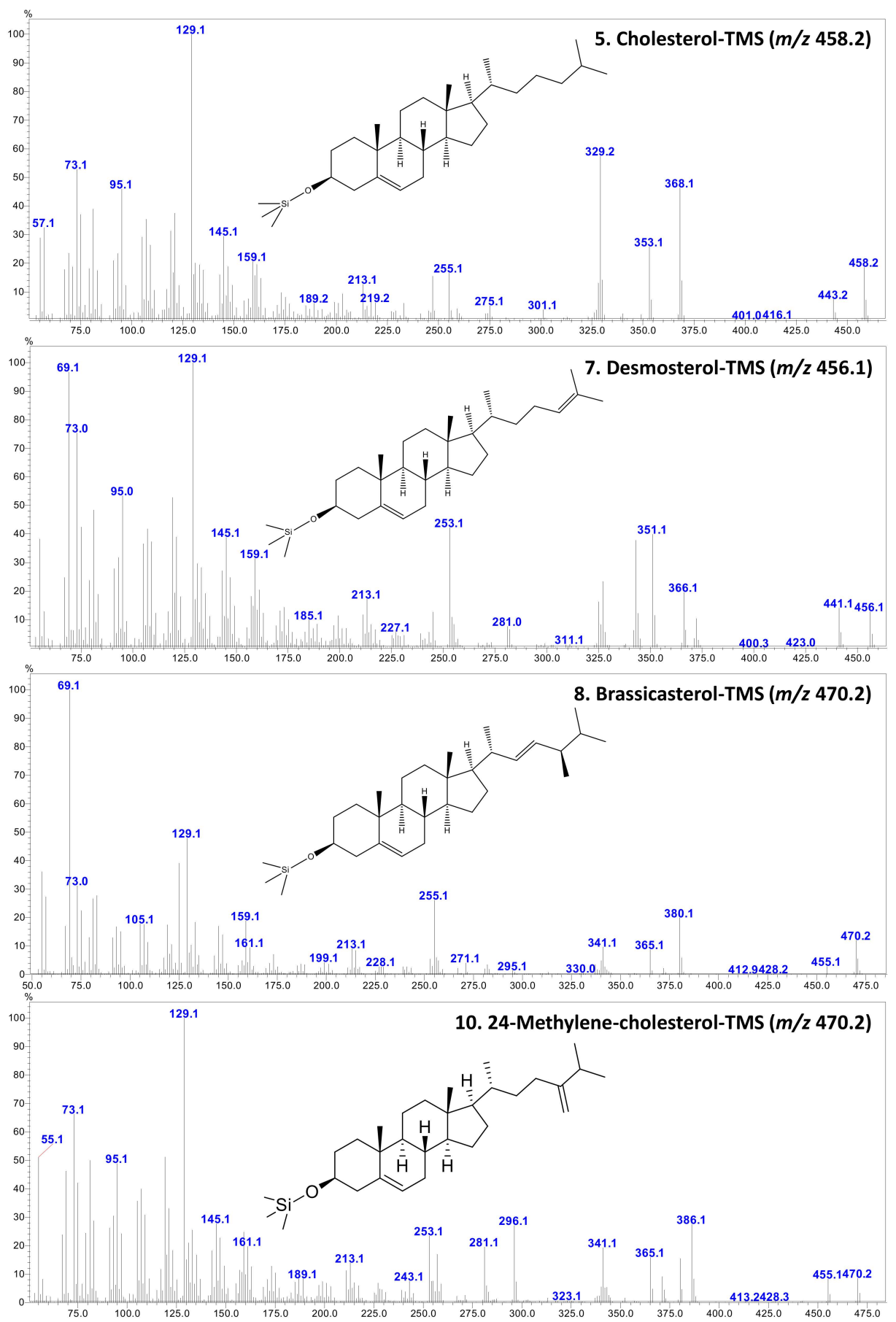

Figure 3. A representative mass spectrometric fragmentation pattern of major sterols (trimethylsiloxy [TMS] derivatives) identified and quantified from the shellfish species. The numbers 5, 7, 10, and 10 correspond to peak numbers in Table 2 and Figure 1. 
Table 3. Sterol composition of shellfish species.

\begin{tabular}{|c|c|c|c|c|c|c|c|c|c|c|c|c|}
\hline Peak No. & Sterol & S1 & S2 & S3 & S4 & S5 & S6 & S7 & S8 & S9 & S10 & S11 \\
\hline 1 & norcholestadienol & $3.30 \pm 0.26$ & $2.85 \pm 0.18$ & $4.03 \pm 0.23$ & $5.17 \pm 0.26^{\mathrm{a}}$ & $4.29 \pm 0.15$ & $0.68 \pm 0.02$ & $0.54 \pm 0.02$ & n.d. & $0.42 \pm 0.04$ & $5.08 \pm 0.09$ a & $0.39 \pm 0.04$ \\
\hline 3 & occelasterol & $0.68 \pm 0.02$ & $0.94 \pm 0.07$ & $1.88 \pm 0.09$ & $2.54 \pm 0.11^{\mathrm{a}}$ & $1.07 \pm 0.03$ & n.d. & n.d. & n.d. & n.d. & $1.64 \pm 0.03$ & n.d. \\
\hline 4 & 22-dehydrocholesterol & $6.23 \pm 0.74$ & $7.15 \pm 0.55$ & $11.3 \pm 0.67^{\mathrm{a}}$ & $8.93 \pm 0.47$ & $7.77 \pm 0.34$ & $3.48 \pm 0.11$ & $3.05 \pm 0.04$ & $0.30 \pm 0.01$ & $3.34 \pm 0.10$ & $9.9 \pm 0.28$ & $1.76 \pm 0.02$ \\
\hline 5 & cholesterol & $38.12 \pm 4.60$ & $67.8 \pm 7.89$ & $32.9 \pm 2.57$ & $32.2 \pm 2.12$ & $33.2 \pm 1.90$ & $65.6 \pm 3.32$ & $71.6 \pm 3.07$ & $107.0 \pm 4.51$ & $154.4 \pm 7.42^{\mathrm{a}}$ & $34.1 \pm 0.95$ & $110.3 \pm 3.70$ \\
\hline 6 & cholestanol & $1.18 \pm 0.12$ & $0.50 \pm 0.00$ & $0.64 \pm 0.02$ & $1.52 \pm 0.08$ & n.d. & n.d. & $0.62 \pm 0.10$ & $1.03 \pm 0.01$ & $0.68 \pm 0.03$ & $2.53 \pm 0.06^{\mathrm{a}}$ & $1.17 \pm 0.06$ \\
\hline 7 & desmosterol & $19.4 \pm 1.78^{a}$ & $14.7 \pm 1.60$ & $6.11 \pm 0.56$ & $1.93 \pm 0.31$ & $1.32 \pm 0.25$ & $1.54 \pm 0.02$ & $2.72 \pm 0.02$ & $3.87 \pm 0.25$ & $16.8 \pm 0.29$ & $6.22 \pm 0.16$ & $1.07 \pm 0.06$ \\
\hline 8 & brassicasterol & $5.04 \pm 0.61$ & $6.88 \pm 0.70$ & $22.6 \pm 1.23^{\mathrm{a}}$ & $11.5 \pm 0.65$ & $12.8 \pm 0.29$ & n.d. & n.d. & n.d. & n.d. & $15.9 \pm 3.38$ & n.d. \\
\hline 9 & ergosterol & $2.92 \pm 0.15$ & $3.43 \pm 0.42$ & $5.26 \pm 0.38^{\mathrm{a}}$ & $0.74 \pm 0.14$ & $1.25 \pm 0.09$ & n.d. & n.d. & n.d. & n.d. & n.d. & n.d. \\
\hline 10 & 24-methylene-cholesterol & $7.63 \pm 0.56$ & $6.61 \pm 0.55$ & $17.2 \pm 1.26$ & $22.7 \pm 0.72^{\mathrm{a}}$ & $19.2 \pm 1.18$ & $2.22 \pm 0.11$ & $1.16 \pm 0.02$ & $1.72 \pm 0.02$ & $1.04 \pm 0.02$ & $17.9 \pm 0.66$ & $1.61 \pm 0.34$ \\
\hline 11 & campesterol & $0.87 \pm 0.01$ & $6.25 \pm 0.41^{\mathrm{a}}$ & $4.85 \pm 0.31$ & $2.19 \pm 0.41$ & $6.35 \pm 0.10^{\mathrm{a}}$ & $1.23 \pm 0.07$ & $0.57 \pm 0.03$ & $0.78 \pm 0.17$ & $\begin{array}{l}\text { n.d. } \\
\text { n. }\end{array}$ & $2.53 \pm 0.74$ & 0.00 \\
\hline 12 & poriferasterol & $0.58 \pm 0.06$ & $0.60 \pm 0.01$ & $1.63 \pm 0.11$ & $0.63 \pm 0.03$ & $1.89 \pm 0.07^{\mathrm{a}}$ & n.d. & n.d. & n.d. & n.d. & $0.95 \pm 0.07$ & n.d. \\
\hline 14 & unidentified 2 & n.d. & $2.12 \pm 0.36^{\mathrm{a}}$ & $0.88 \pm 0.06$ & $\begin{array}{l}\text { n.d. } \\
\text { no }\end{array}$ & n.d. & n.d. & n.d. & n.d. & n.d. & 0.00 & n.d. \\
\hline 15 & clionasterol & $2.34 \pm 0.14$ & $4.59 \pm 0.30$ & $8.48 \pm 0.33^{\mathrm{a}}$ & $3.84 \pm 0.18$ & $7.64 \pm 0.11$ & $0.59 \pm 0.01$ & 0.00 & $0.82 \pm 0.04$ & n.d. & $5.88 \pm 0.17$ & n.d. \\
\hline 16 & isofucosterol & $1.17 \pm 0.01$ & $2.56 \pm 0.17$ & $10.6 \pm 0.56$ & $4.14 \pm 0.19$ & $12.7 \pm 0.43^{\mathrm{a}}$ & $0.22 \pm 0.05$ & n.d. & $\begin{array}{l}\text { n.d. } \\
\text { not }\end{array}$ & n.d. & $5.65 \pm 0.15$ & n.d. \\
\hline 17 & unidentified 3 & $0.83 \pm 0.01$ & $1.93 \pm 0.30^{\mathrm{a}}$ & $1.85 \pm 0.15^{\mathrm{a}}$ & n.d. & $1.39 \pm 0.11$ & n.d. & n.d. & n.d. & n.d. & n.d. & n.d. \\
\hline \multirow[t]{4}{*}{18} & unidentified 4 & n.d. & $0.42 \pm 0.05$ & $1.01 \pm 0.06^{\mathrm{a}}$ & n.d. & $0.87 \pm 0.05$ & n.d. & n.d. & n.d. & n.d. & n.d. & n.d. \\
\hline & total sterols & $140.4 \pm 12.9$ & $172.2 \pm 16.2^{a}$ & $192.3 \pm 12.1^{\mathrm{a}}$ & $176.1 \pm 8.26^{\mathrm{a}}$ & $176.2 \pm 6.84$ a & $85.8 \pm 3.05$ & $88.3 \pm 2.62$ & $115.5 \pm 4.93$ & $182.9 \pm 8.42^{\mathrm{a}}$ & $185.9 \pm 0.70^{\mathrm{a}}$ & $122.2 \pm 4.07$ \\
\hline & total non-cholesterol sterols & $102.3 \pm 8.31$ & $104.3 \pm 8.31$ & $159.4 \pm 9.51^{\mathrm{a}}$ & $144.0 \pm 6.15$ & $143.0 \pm 4.95$ & $20.2 \pm 0.27$ & $16.8 \pm 0.45$ & $8.52 \pm 0.41$ & $28.6 \pm 1.00$ & $151.8 \pm 0.25$ & $11.9 \pm 0.36$ \\
\hline & $\%$ non-cholesterol sterols & $72.9 \pm 0.79$ & $60.7 \pm 0.89$ & $82.9 \pm 0.26^{\mathrm{a}}$ & $81.7 \pm 0.35^{\mathrm{a}}$ & $81.2 \pm 0.35^{\mathrm{a}}$ & $23.6 \pm 1.15$ & $19.0 \pm 1.08$ & $7.37 \pm 0.04$ & $15.6 \pm 0.17$ & $81.6 \pm 0.44^{\mathrm{a}}$ & $9.7 \pm 0.03$ \\
\hline
\end{tabular}

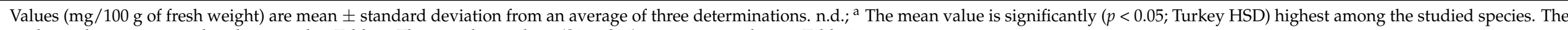

peak numbers correspond to those used in Table 2. The sample numbers (S1 to S11) are correspondent to Table 1.

Table 4. Fatty acid composition of shellfish species.

\begin{tabular}{|c|c|c|c|c|c|c|c|c|c|c|c|c|c|}
\hline Peak No. & FAME & $\mathrm{RT}$ & s1 & s2 & s3 & S4 & s5 & S6 & S7 & s8 & s9 & S10 & S11 \\
\hline 1 & C14:0 (myristic) & 16.91 & $5.81 \pm 0.34$ & $4.36 \pm 0.14$ & $2.02 \pm 0.20$ & $5.36 \pm 0.00$ & $3.28 \pm 0.10$ & $2.59 \pm 0.01$ & $1.84 \pm 0.01$ & $8.77 \pm 0.00^{a}$ & $2.03 \pm 0.00$ & $4.21 \pm 0.02$ & $2.35 \pm 0.11$ \\
\hline 2 & C15:0 (pentadecanoic) & 18.72 & $0.58 \pm 0.01^{\mathrm{a}}$ & $0.75 \pm 0.00^{\mathrm{a}}$ & $0.53 \pm 0.02^{\mathrm{a}}$ & $0.47 \pm 0.00^{a}$ & $0.50 \pm 0.00^{\mathrm{a}}$ & $0.75 \pm 0.0 \mathrm{o}^{\mathrm{a}}$ & $1.12 \pm 1.00 \mathrm{a}$ & $0.76 \pm 0.01 \mathrm{a}$ & $0.42 \pm 0.00^{\mathrm{a}}$ & $0.58 \pm 0.00^{\mathrm{a}}$ & $0.69 \pm 0.09 \mathrm{a}$ \\
\hline 3 & C16:0 (palmitic) & 20.56 & $17.3 \pm 0.20$ & $12.5 \pm 0.24$ & $16.4 \pm 0.04$ & $14.16 \pm 0.05$ & $19.8 \pm 0.04$ & $14.53 \pm 0.05$ & $18.1 \pm 0.20$ & $18.9 \pm 0.05$ & $29.3 \pm 0.09 \mathrm{a}$ & $18.2 \pm 0.00$ & $17.7 \pm 0.27$ \\
\hline 4 & $\begin{array}{l}\text { C16:1 (palmitoleic) } \\
\text { Cit. }\end{array}$ & $\begin{array}{l}21.84 \\
22.34\end{array}$ & $\begin{array}{l}8.73 \pm 0.04 \\
100002\end{array}$ & $\begin{array}{l}5.91 \pm 0.05 \\
0.98\end{array}$ & $7.47 \pm 0.05$ & $6.16 \pm 0.26$ & & $8.45 \pm 0.00$ & $\begin{array}{l}1.18 \pm 0.10 \\
1.6 \pm 0.02\end{array}$ & $1.60 \pm 0.03$ & $0.45 \pm 0.00$ & $3.81 \pm 0.12$ & $4.34 \pm 0.05$ \\
\hline $\begin{array}{l}5 \\
6\end{array}$ & $\begin{array}{l}\text { C17:0 (heptadecanoic) } \\
\text { C18:0 (tearic) }\end{array}$ & $\begin{array}{l}22.34 \\
24.12\end{array}$ & $\begin{array}{l}1.00 \pm 0.02 \\
5.50 \pm 0.05\end{array}$ & $\begin{array}{l}0.98 \pm 0.06 \\
7.8600 .07\end{array}$ & $\begin{array}{l}1.33 \pm 0.01 \\
7.65+0.06\end{array}$ & $\begin{array}{l}0.51 \pm 0.06 \\
5.95 \pm 0.00\end{array}$ & $\begin{array}{l}1.49 \pm 0.01 \\
5.55 \pm 0.23\end{array}$ & $\begin{array}{l}0.88 \pm 0.01 \\
5.68 \pm 0.00\end{array}$ & $\begin{array}{l}1.63 \pm 0.02 \mathrm{a}^{2} \\
8.92 \pm 0.11\end{array}$ & $\begin{array}{l}0.57 \pm 0.00 \\
5.79 \pm 0.02\end{array}$ & $\begin{array}{l}0.67 \pm 0.00 \\
5.2220 .01\end{array}$ & $\begin{array}{l}1.0290 .04 \\
4.5 \pm 0.07\end{array}$ & $\begin{array}{l}0.95 \pm 0.07 \\
5.05 \pm 0.04\end{array}$ \\
\hline $\begin{array}{l}6 \\
7\end{array}$ & $\begin{array}{l}\text { C18:0 (staearic) } \\
\text { C18:1n9c (oleic) }\end{array}$ & $\begin{array}{l}24.12 \\
25.19\end{array}$ & $\begin{array}{l}5.50 \pm 0.05 \\
1.09 \pm 0.02\end{array}$ & $\begin{array}{l}7.860 \pm 0.07 \\
1.84 \pm 0.05\end{array}$ & $\begin{array}{l}7.65 \pm 0.06 \\
2.62 \pm 0.02\end{array}$ & $\begin{array}{l}5.95 \pm 0.000 \\
2.20 \pm 0.24\end{array}$ & $\begin{array}{l}5.85 \pm 0.23 \\
2.96 \pm 0.02\end{array}$ & $\begin{array}{l}5.68 \pm \pm 0.00 \\
15.21 \pm 0.02\end{array}$ & $\begin{array}{l}8.92 \pm 0.111^{\mathrm{a}} \\
2.41 \pm 0.04\end{array}$ & $\begin{array}{l}5.79 \pm 0.02 \\
5.69 \pm 0.04\end{array}$ & $\begin{array}{l}5.222 \pm 0.01 \\
1.54 \pm 0.01\end{array}$ & $\begin{array}{l}4.5990 .07 \\
1.71 \pm 0.03\end{array}$ & $\begin{array}{r}5.05 \pm 0.04 \\
16.49 \pm 0.20 \mathrm{a}\end{array}$ \\
\hline 8 & 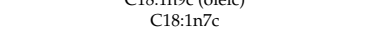 & 25.34 & $3.10 \pm 0.01$ & $\begin{array}{l}1.040 .05 \\
2.21 \pm 0.02\end{array}$ & $3.45 \pm 0.02$ & $\begin{array}{l}3.203 \pm 0.01 \\
3.93\end{array}$ & $2.94 \pm 0.01$ & $5.49 \pm 0.01$ & $\begin{array}{l}2.41 \pm 0.04 \\
2.85 \pm 0.02\end{array}$ & $9.42 \pm 0.00 \mathrm{a}$ & $\begin{array}{l}1.040 .01 \\
1.39 \pm 0.00\end{array}$ & $5.26 \pm 0.02$ & $\begin{array}{l}30.71 \pm 0.06 \\
3.71\end{array}$ \\
\hline 9 & C18:2n6c (linoleic) & 26.81 & $1.62 \pm 0.01$ & $3.19 \pm 0.15 \mathrm{a}$ & $0.61 \pm 0.01$ & $2.07 \pm 0.02$ & $0.78 \pm 0.01$ & $1.43 \pm 0.01$ & $0.40 \pm 0.12$ & $2.58 \pm 0.02$ & $0.32 \pm 0.02$ & $1.30 \pm 0.02$ & $1.68 \pm 0.15$ \\
\hline 10 & C20:1n9 (cis-11-eicosenoic) & 28.33 & $2.06 \pm 0.00$ & $4.36 \pm 0.03 \mathrm{a}$ & $3.00 \pm 0.03$ & $1.76 \pm 0.02$ & $2.20 \pm 0.05$ & $1.84 \pm 0.00$ & $0.50 \pm 0.03$ & $3.81 \pm 0.00$ & $0.16 \pm 0.01$ & $1.83 \pm 0.05$ & $0.38 \pm 0.10$ \\
\hline${ }_{11}^{11}$ & $\begin{array}{c}\text { C20:1n7c } \\
\text {. }\end{array}$ & $\begin{array}{l}28.47 \\
28.63\end{array}$ & $3.25 \pm 0.00$ & $\begin{array}{l}2.73 \pm 0.01 \\
2766+01\end{array}$ & $\begin{array}{l}1.55 \pm 0.01 \\
272+0.03\end{array}$ & $1.94 \pm 0.01$ & $1.57 \pm 0.05$ & $1.56 \pm 0.02$ & $\begin{array}{l}4.85 \pm 0.00^{\mathrm{a}} \\
.058+0.5\end{array}$ & $1.05 \pm 0.01$ & $\begin{array}{l}2.74 \pm 0.02 \\
0.017+0.1\end{array}$ & $\begin{array}{r}0.64 \pm 0.05 \\
5.85+00^{\mathrm{a}}\end{array}$ & $1.06 \pm 0.00$ \\
\hline 12 & $\begin{array}{l}\text { C18:3333 ( } \alpha \text {-linolenic) } \\
\text { C18:4n3 (stearidonic) }\end{array}$ & $\begin{array}{l}28.63 \\
29.85\end{array}$ & $\begin{array}{l}2.511 \pm 0.01 \\
1.60 \pm 0.00\end{array}$ & $\begin{array}{l}2.76 \pm 0.01 \\
0.99 \pm 0.01\end{array}$ & $\begin{array}{l}2.72 \pm 0.03 \\
1.35 \pm 0.00\end{array}$ & $\begin{array}{l}1.87 \pm 0.02 \\
1.33 \pm 0.01\end{array}$ & $\begin{array}{l}5.05 \pm 0.06 \\
1.74 \pm 0.00\end{array}$ & $\begin{array}{l}1.55 \pm 0.00 \\
0.54 \pm 0.00\end{array}$ & $\begin{array}{l}0.05 \pm 0.0 .05 \\
0.07 \pm 0.01\end{array}$ & $\begin{array}{l}2.37 \pm 0.01 \\
0.85 \pm 0.00\end{array}$ & $\begin{array}{l}0.177 \pm 0.01 \\
0.06 \pm 0.00\end{array}$ & $\begin{array}{l}5.85 \pm 0.0 .4 \mathrm{a}^{\mathrm{a}} \\
2.60 \pm 0.0 \mathrm{a}^{2}\end{array}$ & $\begin{array}{l}1.32 \pm 0.01 \\
0.56 \pm 0.01\end{array}$ \\
\hline 14 & C20:2n6 (cis-11,14-eicosadienoic) & 29.98 & $\begin{array}{l}1.00 \pm 0.00 \\
0.46 \pm 0.01\end{array}$ & $\begin{array}{l}0.99 \pm 0.01 \mathrm{a} \\
2.53 \pm 0.01 \mathrm{a}\end{array}$ & $\begin{array}{l}1.50 \pm 0.00 \\
1.60 \pm 0.01\end{array}$ & $\begin{array}{l}1.33 \pm 0.01 \\
0.66 \pm 0.01\end{array}$ & $\begin{array}{l}1.14 \pm 0.00 \\
1.60 \pm 0.00\end{array}$ & $\begin{array}{l}0.04 \pm 0.00 \\
1.12 \pm 0.01\end{array}$ & $\begin{array}{l}0.57 \pm 0.01 \\
0.52 \pm 0.04\end{array}$ & $\begin{array}{l}0.43 \pm 0.00 \\
0.48 \pm 0.01\end{array}$ & $\begin{array}{l}0.000 \pm 0.00 \\
0.21 \pm 0.01\end{array}$ & $\begin{array}{l}2.00 \pm 0.000 \\
0.28 \pm 0.05\end{array}$ & $\begin{array}{l}0.03 \pm 0.01 \\
0.94 \pm 0.04\end{array}$ \\
\hline 15 & C20:4n6 (arachidonic) & 32.03 & $4.73 \pm 0.02$ & $10.79 \pm 0.00$ & $4.01 \pm 0.03$ & $3.18 \pm 0.03$ & $5.42 \pm 0.02$ & $5.83 \pm 0.00$ & $7.63 \pm 0.04$ & $16.37 \pm 0.02 \mathrm{a}$ & $2.42 \pm 0.00$ & $3.52 \pm 0.08$ & $3.04 \pm 0.16$ \\
\hline \multirow{5}{*}{18} & C22:5n3 (cis-7, 10,13,16,19-docosapentaenoic) & 37.81 & $1.63 \pm 0.05$ & $9.10 \pm 0.02 \mathrm{a}$ & $2.79 \pm 0.01$ & $0.75 \pm 0.01$ & $3.37 \pm 0.02$ & $2.02 \pm 0.01$ & $2.33 \pm 0.03$ & $9.16 \pm 0.02^{\mathrm{a}}$ & $0.47 \pm 0.00$ & $1.78 \pm 0.01$ & $0.88 \pm 0.05$ \\
\hline & $\begin{array}{l}\text { C22:6n3 (cis-4,7,10,13,16,19-docosahexaenoic) } \\
\Gamma \text { SFAs }\end{array}$ & 38.93 & $\begin{array}{l}16.5 \pm 0.04 \\
30.1+0.06\end{array}$ & $\begin{array}{l}11.5 \pm 0.01 \\
265+0.11\end{array}$ & $\begin{array}{l}28.4 \pm 0.12 \\
280+0.22\end{array}$ & $\begin{array}{l}15.9 \pm 0.10 \\
26.4+0.11\end{array}$ & $\begin{array}{l}16.0 \pm 0.04 \\
30.9 \pm 0.09\end{array}$ & $\begin{array}{l}17.1 \pm 0.01 \\
24.4 \pm 0.03\end{array}$ & $\begin{array}{l}25.8 \pm 0.20 \\
31.6 \pm 0.69\end{array}$ & $\begin{array}{l}\text { n.d. } \\
34.8+0.08\end{array}$ & $\begin{array}{l}39.6 \pm 0.022^{\mathrm{a}} \\
376 \pm 0.08 \mathrm{a}\end{array}$ & $\begin{array}{l}17.99+0.05 \\
286+0.01\end{array}$ & $\begin{array}{l}21.0 \pm 1.26 \\
26.7 \pm 0.56\end{array}$ \\
\hline & $\begin{array}{l}\text { LMUAS } \\
\text { ¿MUFAs }\end{array}$ & & $\begin{array}{l}30.1 \pm 0.06 \\
18.2 \pm 0.07\end{array}$ & $\begin{array}{l}26.900 .011 \\
17.0 \pm 0.06\end{array}$ & $\begin{array}{l}28.0 \pm 0.22 \\
18.1 \pm 0.01\end{array}$ & $\begin{array}{l}26.4 \pm 0.11 \\
16.0 \pm 0.54\end{array}$ & $\begin{array}{l}30.9 \pm 0.09 \\
19.84 \pm 0.03\end{array}$ & $\begin{array}{l}24.4 \pm 0.03 \\
32.5 \pm 0.01\end{array}$ & $\begin{array}{l}31.6 \pm 0.69 \\
11.8 \pm 0.13\end{array}$ & $\begin{array}{l}34.8 \pm 0.08 \\
21.6 \pm 0.07\end{array}$ & $\begin{aligned} & 37.6 \pm 0.008 \\
& 6.29 \pm 0.00\end{aligned}$ & $\begin{array}{l}28.0 \pm 0.001 \\
13.2 \pm 0.12\end{array}$ & $\begin{array}{l}26.7 \pm 0.06 \\
26.0 \pm 0.21\end{array}$ \\
\hline & $\sum$ PUFAs & & $51.6 \pm 0.01$ & $56.5 \pm 0.18$ & $53.9 \pm 0.24$ & $57.6 \pm 0.64$ & $49.28 \pm 0.06$ & $43.0 \pm 0.02$ & $56.6 \pm 0.56$ & $43.6 \pm 0.15$ & $56.1 \pm 0.08$ & $58.1 \pm 0.11^{\mathrm{a}}$ & $47.3 \pm 0.77$ \\
\hline & crude lipids $(\% \mathrm{FW})$ & & $2.88 \pm 0.17$ & $2.79 \pm 0.29$ & $2.37 \pm 0.18$ & $2.75 \pm 0.35$ & $2.78 \pm 0.32$ & $4.29 \pm 0.42^{\mathrm{a}}$ & $1.37 \pm 0.18$ & $1.90 \pm 0.14$ & $3.32 \pm 0.26$ & $3.90 \pm 0.14^{\mathrm{a}}$ & $1.91 \pm 0.13$ \\
\hline
\end{tabular}

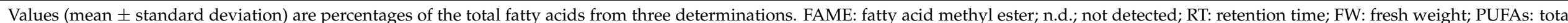

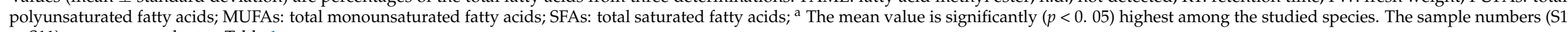
to S11) are correspondent to Table 1. 


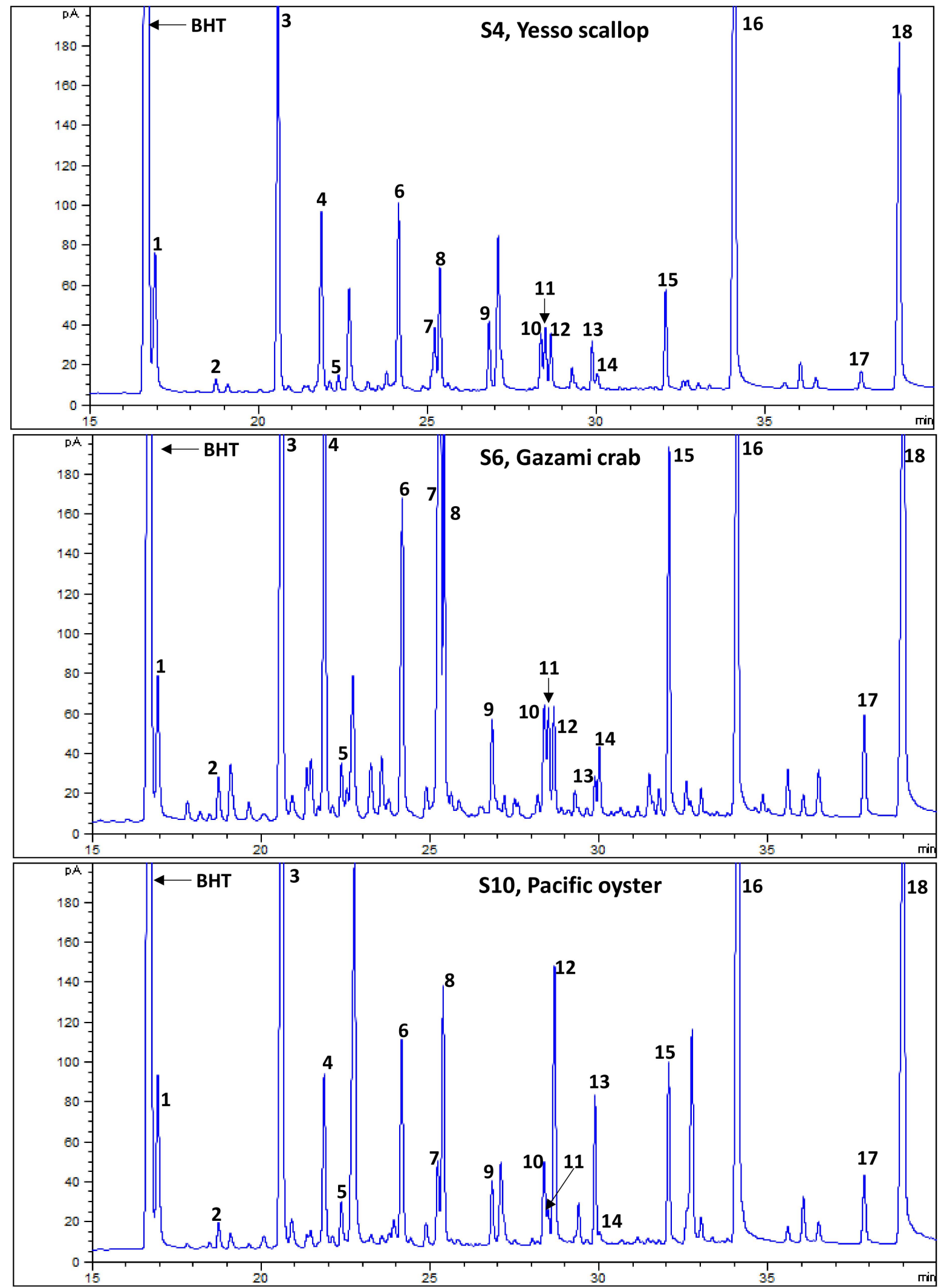

Figure 4. The representative gas chromatography (GC) flame ionization detection (FID) chromatograms of fatty acids and methyl esters (FAMEs) of the Yesso scallop, Gazami crab, and oyster. The peak numbers (1 to 18) correspond to Table 4. 
With the presence of the highest amount of $\alpha$-linolenic (C18:3n3; 5.85\%), stearidonic (C18:4n3; $2.60 \%)$, and a fairly good amount of EPA $(24.9 \%)$, and DHA $(17.9 \%)$, the highest amount of total PUFAs (58.1\% of total fatty acids) were recorded from the Pacific oyster. The representative GC-FID chromatograms of arrow squid and pacific oyster are given in Figure 4.

The lipid composition recorded in the present investigation are in agreement with previous studies on the far eastern mussel [40], Japanese littleneck [14], Yesso scallop [14], abalone [17], and Argentine red shrimp [22]. However, contrasting results were obtained for some shellfish species. For instance, Saito and Aono [19] recorded only a trace amount of DHA in the different lipid fractions of wild and cultured horned turban viscera. However, in the present investigation, we recorded a significant amount of DHA $(11.5 \%)$ in the horned turban. Similarly, Lu et al. [18] recorded a significantly higher amount of EPA and DHA in the edible viscera of female Gazami crab (7.72\% EPA and 16.5\% DHA), compared with the male Gazami crab (3.47\% EPA and 7.30\% DHA). We recorded $13.4 \%$ EPA and $17.1 \%$ DHA in the male Gazami crab (female Gazami crab not investigated) in the present investigation.

Fatty acid compositions in seafood may vary with season [17], sex (e.g., male vs. female crab) [18], culturing conditions (e.g., wild vs. cultured, diet quality) $[19,20]$, and several other factors [21].

Shellfish species are well known to contain a significant amount of EPA, DHA, and other health-beneficial n-3 fatty acids [41-43]. In the present study, all the studied shellfish showed the presence of a significant amount of DHA, except abalone. Previous studies have also shown the complete absence of DHA in abalone species [17]. In the present study, abalone showed the unusual presence of the highest amount of docosapentaenoic acid (C22:5n3); a most abundant n-3 VLC-PUFA in the human brain after DHA, beneficial for elderly neuroprotection and early-life development [44].

\subsection{Fatty Acid Indices}

The consumption of PUFAs, especially n-3 PUFAs in appropriate proportions, is highly beneficial in reducing risk to CVD and many other chronic diseases [45,46]. Among the studied species, the highest amount of total n-3 PUFAs were recorded from arrow squid $(53.2 \%)$ and pacific oyster (53.0\%) (Table 5). However, with the presence of the highest amount of palmitic acid (29.3\% of total lipids), the highest total SFAs $(37.6 \%)$ were also recorded in arrow squid. In view of the risk of CVD and other chronic diseases associated with the consumption of SFAs [46], fats with PUFAs/SFAs ratios of greater than 0.45 are considered safe for human consumption [28]. In the present study, the PUFAs/SFAs ratios ranged from 1.25 (abalone) to 2.18 (Yesso scallop) (Table 5), showing that lipids from all the studied shellfish species are healthy for consumption. Furthermore, the fats with higher ratios of $\mathrm{h} / \mathrm{H}$ fatty acids and lower $\mathrm{TI}$ and $\mathrm{AI}$, are suggested for minimizing the risk of CVD [27]. In the present study, a significant difference was recorded for $\mathrm{h} / \mathrm{H}$ fatty acid ratios, TI and AI values of fat obtained from the shellfish species. The significantly highest $\mathrm{h} / \mathrm{H}$ fatty acid ratios of 4.41 and 4.36 were recorded from Gazami crab and Yesso scallop, respectively. Similarly, the lowest TI (0.15) and AI (0.33) were also recorded also recoded from the Yesso scallop, and Gazmi crab, respectively. These observations indicate the lipids obtained from the Yesso scallop and Gazmi crab are least hypercholesteromic, atherogenic and thrombogenic, hypercholestromic. 
Table 5. Fat quality indices of lipids obtained from shellfish species.

\begin{tabular}{|c|c|c|c|c|c|c|c|c|c|c|c|}
\hline Components & S1 & S2 & S3 & S4 & S5 & S6 & S7 & S8 & S9 & S10 & S11 \\
\hline PUFAs: SFAs & $1.71 \pm 0.00$ & $2.13 \pm 0.02$ & $1.93 \pm 0.02$ & $2.18 \pm 0.03 \mathrm{a}$ & $1.60 \pm 0.01$ & $1.76 \pm 0.00$ & $1.79 \pm 0.06$ & $1.25 \pm 0.01$ & $1.49 \pm 0.01$ & $2.03 \pm 0.00$ & $1.77 \pm 0.07$ \\
\hline$\sum \mathrm{n}-3$ PUFAs $\#$ & $44.8 \pm 0.01$ & $40.0 \pm 0.01$ & $47.7 \pm 0.19$ & $51.6 \pm 0.70^{\mathrm{a}}$ & $41.5 \pm 0.03$ & $34.6 \pm 0.04$ & $48.0 \pm 0.43$ & $24.2 \pm 0.18$ & $53.2 \pm 0.05^{\mathrm{a}}$ & $53.0 \pm 0.12^{\mathrm{a}}$ & $41.7 \pm 1.12$ \\
\hline$\sum \mathrm{n}-6$ PUFA \# & $6.82 \pm 0.02$ & $16.5 \pm 0.16$ & $6.22 \pm 0.04$ & $5.92 \pm 0.05$ & $7.80 \pm 0.03$ & $8.39 \pm 0.02$ & $8.55 \pm 0.13$ & $19.4 \pm 0.03 \mathrm{a}$ & $2.95 \pm 0.03$ & $5.11 \pm 0.01$ & $5.65 \pm 0.35$ \\
\hline $\mathrm{n}-3 / \mathrm{n}-6$ PUFA & $6.58 \pm 0.02$ & $2.42 \pm 0.02$ & $7.67 \pm 0.02$ & $8.73 \pm 0.20$ & $5.32 \pm 0.02$ & $4.13 \pm 0.01$ & $5.62 \pm 0.03$ & $1.25 \pm 0.01$ & $18.05 \pm 0.15^{a}$ & $10.4 \pm 0.04$ & $7.39 \pm 0.65$ \\
\hline $\mathrm{AI}$ & $0.58 \pm 0.02$ & $0.41 \pm 0.00$ & $0.34 \pm 0.01$ & $0.48 \pm 0.00$ & $0.48 \pm 0.01$ & $0.33 \pm 0.00$ & $0.37 \pm 0.00$ & $0.83 \pm 0.00^{\mathrm{a}}$ & $0.60 \pm 0.00$ & $0.49 \pm 0.00$ & $0.37 \pm 0.01$ \\
\hline TI & $0.19 \pm 0.00$ & $0.18 \pm 0.00$ & $0.16 \pm 0.00$ & $0.15 \pm 0.00$ & $0.20 \pm 0.00$ & $0.18 \pm 0.00$ & $0.18 \pm 0.00$ & $0.35 \pm 0.00^{\mathrm{a}}$ & $0.20 \pm 0.00$ & $0.15 \pm 0.00$ & $0.17 \pm 0.01$ \\
\hline $\mathrm{h} / \mathrm{H}$ & $3.03 \pm 0.02$ & $4.36 \pm 0.03^{a}$ & $3.90 \pm 0.05$ & $3.77 \pm 0.01$ & $3.00 \pm 0.01$ & $4.41 \pm 0.01^{\mathrm{a}}$ & $3.42 \pm 0.00$ & $2.36 \pm 0.01$ & $1.99 \pm 0.01$ & $3.18 \pm 0.00$ & $3.66 \pm 0.10$ \\
\hline
\end{tabular}

\# Values (mean \pm standard deviation) from three determinations. PUFAs: total polyunsaturated fatty acids; MUFAs: total monounsaturated fatty acids; SFAs: total saturated fatty acids; n-3: omega-3; n-6: omega-6; h/H: ratios of hypocholesterolemic (h)/hypercholesterolemic $(\mathrm{H})$ fatty acids; TI: thrombogenic index; and AI: atherogenic index. ${ }^{a}$ The mean value is significantly $(p<0.05)$ highest among the studied species. The sample numbers (S1 to S11) are correspondent to Table 1.

\subsection{Tocols Composition}

This study screened lipids obtained from the shellfish species for tocols composition by HPLC-DAD. A significant amount of $\alpha$-tocopherol was recorded in all studied samples, with the highest content in the Gazami crab $(30.1 \mu \mathrm{g} / \mathrm{g}$ FW) (Figure 5), while other forms were not detected in a substantial amount.

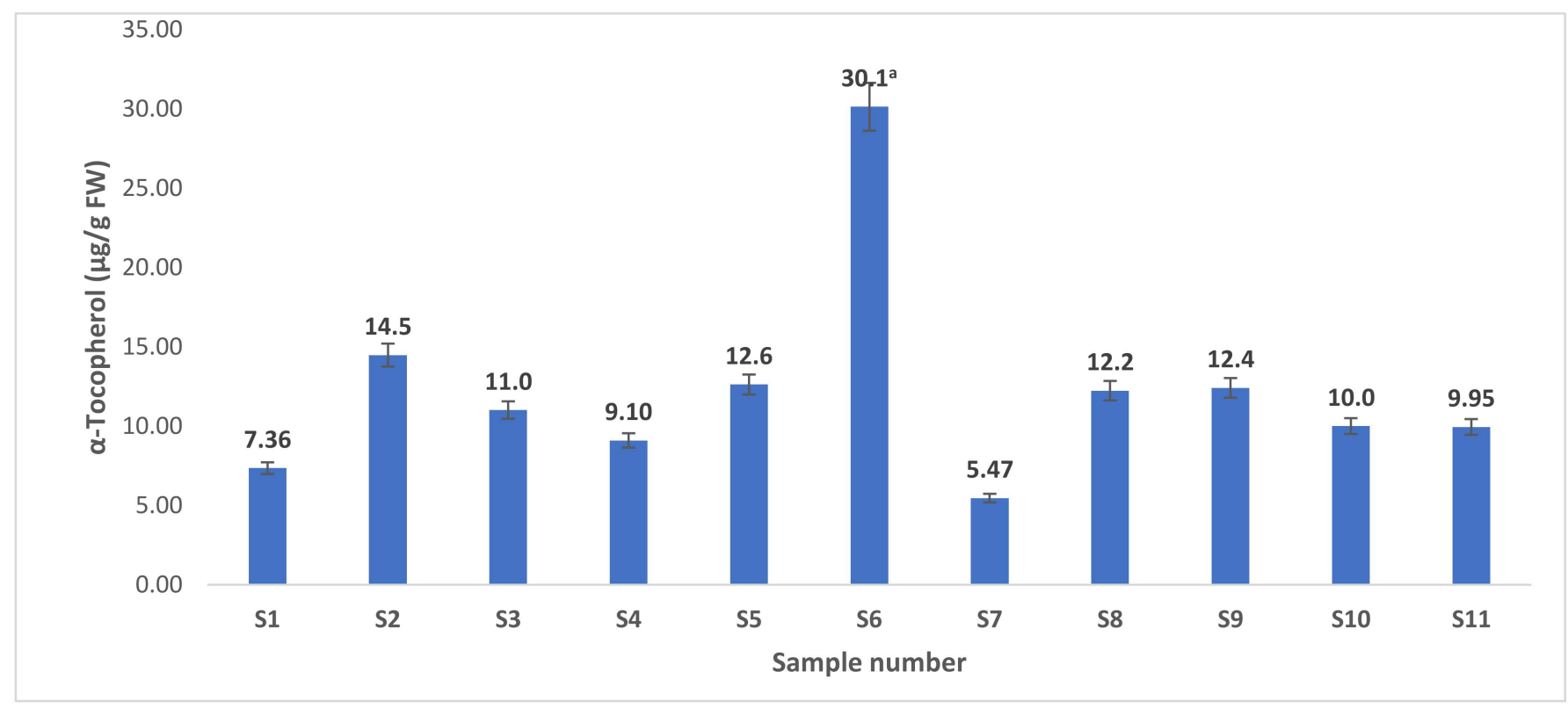

Figure 5. The contents of $\alpha$-tocopherol ( $\mu \mathrm{g} / \mathrm{g}$ fresh weight) of the studied shellfish species. Values are mean \pm standard deviation of three replicates. ${ }^{\text {a }}$ The mean value is significantly $(p<0.05$, Turkey HSD) highest among the studied species. The sample numbers (S1 to S11) correspond to Table 1.

Only a few studies are available on the tocopherol contents of shellfish species. In raw clams (unknown species), Kuhnlein et al. [11] recorded $5.7 \mu \mathrm{g} / \mathrm{g}$ of $\alpha$-tocopherol, However, in the present study, we recorded $12.6 \mu \mathrm{g} / \mathrm{g}$ of $\alpha$-tocopherol in the common orient clam. Tocopherols are potent free radical scavengers that play a crucial role in minimizing oxidative stress-related diseases, including cancer, cardiovascular, and neurodegenerative diseases [11,12]. Thus, a diet rich in tocopherols may provide protection against these chronic diseases.

\subsection{Antioxidant Activity of Lipids Extracted from Shellfish Species}

Results of $\mathrm{DPPH}^{+\bullet}$ - and $\mathrm{ABTS}^{+\bullet}$-scavenging activities of lipids extracted from the shellfish species are shown in Figure 6. Among the studied shellfish species, the highest $\mathrm{ABTS}^{+} \bullet$-scavenging activities of $4.79 \mathrm{mg} \mathrm{TE} / \mathrm{g}$ of lipids were recorded from the far eastern mussel. In contrast, the highest $\mathrm{DPPH}^{+} \bullet$-scavenging activities of $3.53 \mathrm{mg} \mathrm{TE} / \mathrm{g}$ of lipids were recorded from the Gazami crab. Surprisingly, in this study, the highest content $\alpha$-tocopherol was also recorded in the Gazami crab. 


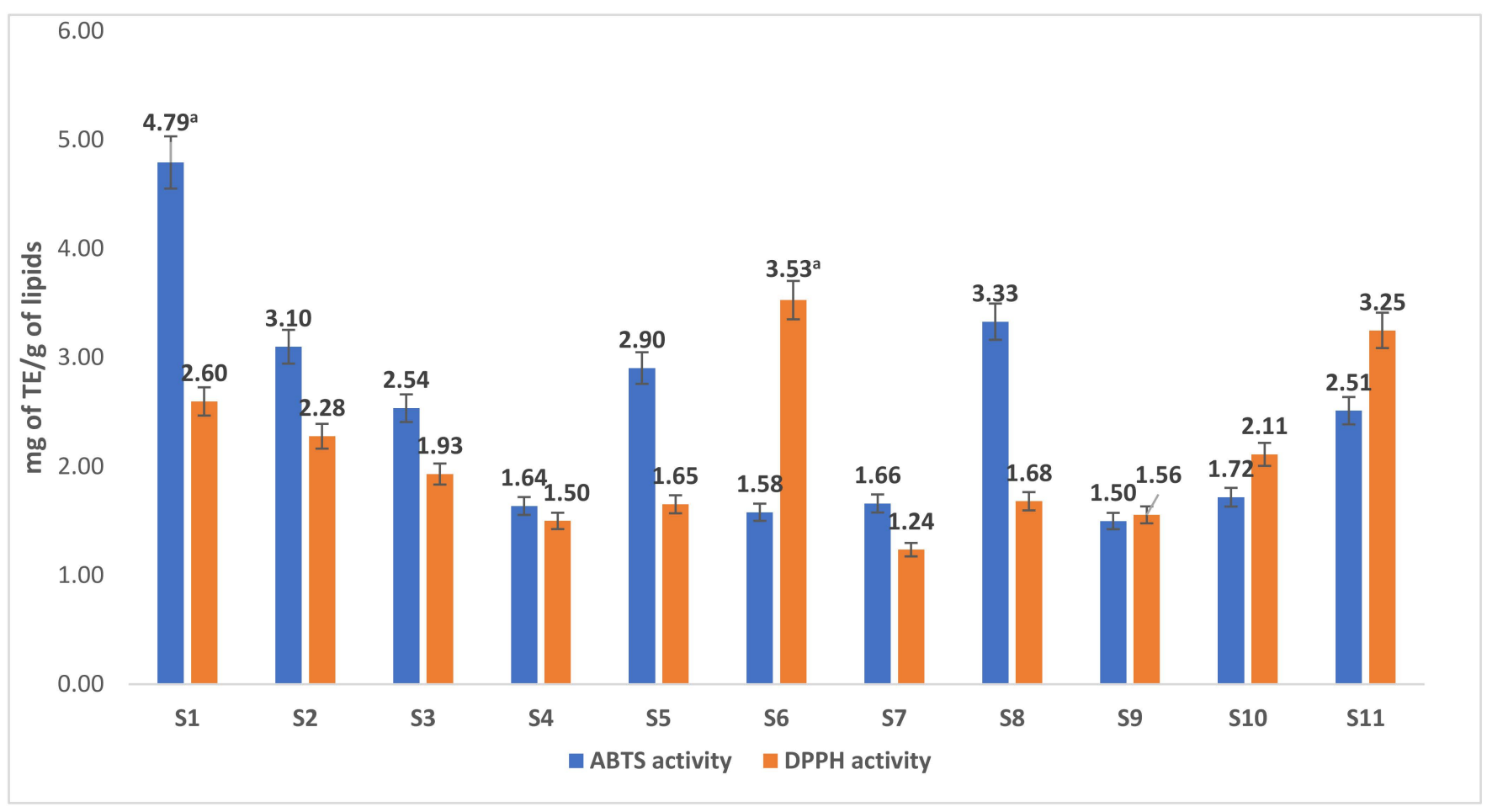

Figure 6. The antioxidant activity (mg of Trolox equivalent (TE)/g of lipids) of studied shellfish species. Values are mean \pm standard deviation of three replicates. ${ }^{\text {a }}$ The mean value is significantly $(p<0$. 05, Turkey HSD) highest among the studied species. The sample numbers (S1 to S11) are correspondent to Table 1.

Although both DPPH and ABTS assays are commonly classified as electron transfer (ET) reactions, these two radicals actually may be deactivated either by direct reduction through ET mechanisms or by radical quenching via hydrogen atom transfer (HAT) [47]. The higher DPPH free radical scavenging activities of the Gazmi crab is probably due to the higher contents of $\alpha$-tocopherol, which is considered a potent free radical scavenger [12]. However, the higher ABTS activities of far eastern mussel (containing a low amount of $\alpha$-tocopherol), suggesting that other lipophilic compounds are probably responsible for these activities. Overall, the strong free radical scavenging activities of shellfish lipids may help in minimizing oxidative stress-related diseases [11,12].

\subsection{Anticancer Potential of Lipids Extracted from Shellfish Species}

The few reports on the effect of shellfish lipids in cancer are controversial. While n-3 PUFAs, the most well-known fatty acids in shellfish lipids, was previously reported to have antitumoral effects [43,44], it was also reported that shellfish intake increased the risk of head and neck cancer [45].

The lipophilic compounds recorded in the present investigation, such as $\alpha$ tocopherol [48], n-3 PUFAs (especially EPA and DHA) [49], and sterols [9], have shown anticancer effects. In the present investigation, the brassicasterol, 24-methylene-cholesterol, 22dehydrocholesterol, and isofucosterol were recorded as the dominant NCSs. Brassicasterolrich lipids from edible Hippocampus abdominalis have shown cytotoxicity against LNCaP human prostate cancer cells [50]. Lipid fractions rich in 22-dehydrocholesterol, brassicasterol and other marine animal specific sterols from the Persian Gulf sponge, Axinella sinoxea, have also shown cytotoxicity against human lymphoblastic leukemia (MOLT-4), human breast adenocarcinoma (MCF-7), and human colorectal adenocarcinoma (HT-29) cells.

In our study, we found that most of the lipids assayed have anticancer activities, while, at low concentrations, specific lipids showa biphasic response, that is, a hormesis effect, for specific cell lines (Figure 7), suggesting that such response is specific to concentration threshold as well as to cancer type. Among the eleven types of studied shellfish, the 
Argentine red shrimp lipid extract was the most cytotoxic lipid across all cancer cells investigated, while the Gazami crab's lipids were relatively less cytotoxic to cancer cells, except for Hela cells, as can be observed from the $\mathrm{IC}_{50}$ obtained (Figure 8).

S1, Far eastern mussel

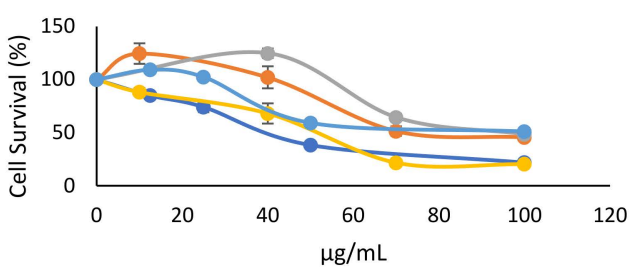

S3, Japanese littleneck

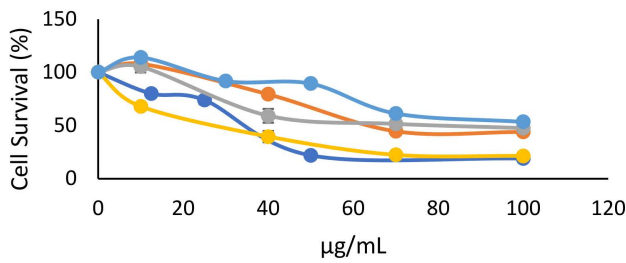

S5, Common orient clam

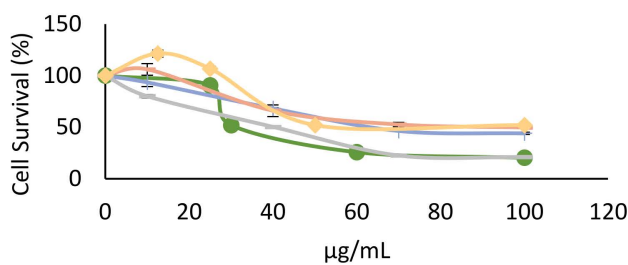

S7, Long arm octopus

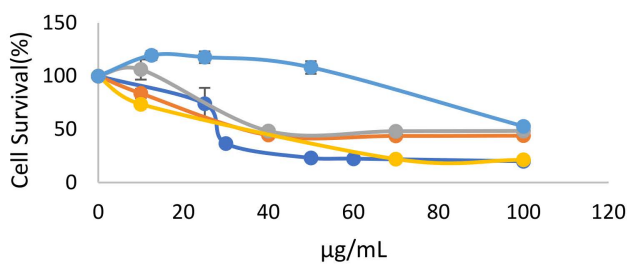

S9, Arrow Squid

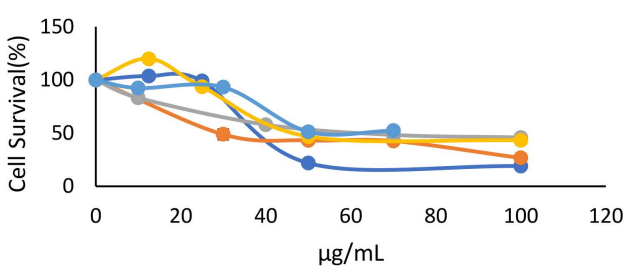

S11, Argentine red shrimp

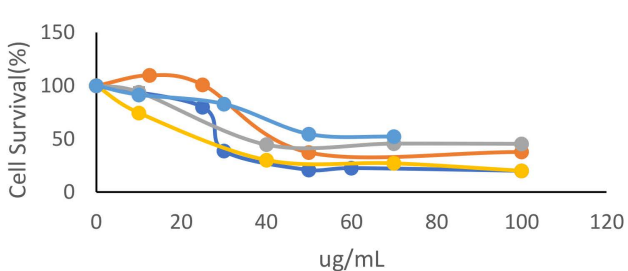

S2, Horned turban

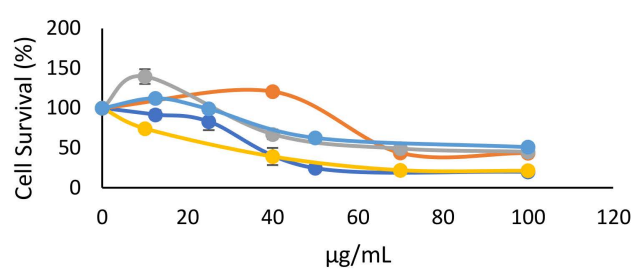

S4, Yesso scallop

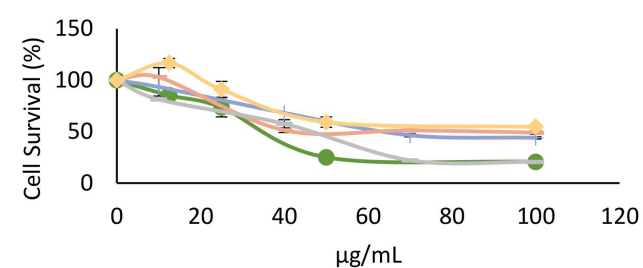

S6, Gazami crab

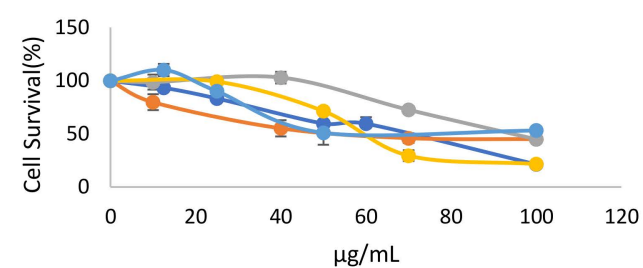

S8, Abalone

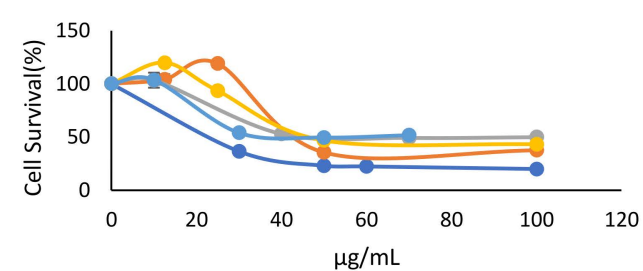

S10, Pacific oyster

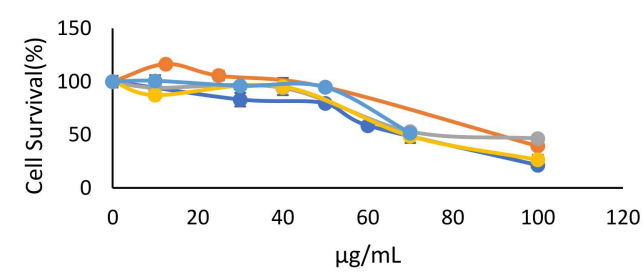

$\longrightarrow$ HCT116

$-\mathrm{A} 2058$

$\multimap-T 98 G$

$\longrightarrow$ A549

$\multimap$ Hela

Figure 7. Cell survival analysis of shellfish lipids treatment to cancer cell lines. Values are mean standard \pm standard deviation of three replicates for cell survival in \% compared to control (0.5\% DMSO) treatment for $24 \mathrm{~h}$. 


\begin{tabular}{|c|c|c|c|c|c|c|}
\hline \multirow[b]{2}{*}{ Sample No. } & \multirow[b]{2}{*}{ Common name } & \multicolumn{5}{|c|}{$\mathrm{IC}_{50}(\mu \mathrm{g} / \mathrm{mL})$} \\
\hline & & HCT116 & A2058 & T98G & A549 & Hela \\
\hline S1 & Far eastern mussel & 36.0 & 50.7 & 66.8 & 43.1 & 39.2 \\
\hline S2 & Horned turban & 32.3 & 58.4 & 34.6 & 21.3 & 38.0 \\
\hline S3 & Japanese littleneck & 30.6 & 41.8 & 34.6 & 20.5 & 58.0 \\
\hline $\mathrm{S} 4$ & Yesso scallop & 30.2 & 38.7 & 28.0 & 40.7 & 28.4 \\
\hline S5 & Common orient clam & 28.9 & 33.9 & 33.5 & 30.7 & 30.3 \\
\hline S6 & Gazami crab & 69.4 & 54.1 & 69.7 & 53.7 & 26.4 \\
\hline S7 & Long arm octopus & 25.2 & 55.2 & 24.6 & 13.3 & 56.9 \\
\hline S8 & Abalone & 20.3 & 36.6 & 33.2 & 27.0 & 25.3 \\
\hline S9 & Arrow squid & 35.4 & 19.7 & 20.4 & 28.1 & 33.3 \\
\hline S10 & Pacific oyster & 62.1 & 57.4 & 56.0 & 67.1 & 54.7 \\
\hline $\mathrm{S} 11$ & Argentine red shrimp & 27.2 & 29.0 & 12.3 & 14.9 & 33.9 \\
\hline
\end{tabular}

Figure 8. IC50 of eleven shellfish extracted lipids. The green, yellow, and red color explains the low, medium, and high cytotoxicity, respectively.

\section{Conclusions}

The results of the present investigation indicated that the contents and composition of sterols, fatty acids, and tocopherols varied significantly among shellfish species. The highest n-3 PUFAs were recorded from arrow squid and pacific oysters, accounting for $53.2 \%$ and $53.0 \%$ of total fatty acids, respectively. However, the highest cholesterol content was recorded in arrow squid (154.4 mg/100 g; 92.6\% of total sterols). In contrast, in the Japanese littleneck, Yesso scallop, and common orient clam, cholesterol was just 17.1\%, $18.3 \%$, and $18.9 \%$ of total sterols, respectively, which makes them the richest source of NCSs. The fat-quality indices indicated that lipids obtained from the Yesso scallop and Gazmi crab are least hypercholesteremic, atherogenic, and thrombogenic.

Lipids extracted from shellfish species showed $\mathrm{ABTS}^{+\bullet}$-and $\mathrm{DPPH}^{+\bullet}$-scavenging activities. The highest $\mathrm{ABTS}^{+\bullet}$-scavenging activities were recorded from the far eastern mussel, while the lipids from the Gazami crab showed the highest $\mathrm{DPPH}^{+\bullet}$-scavenging activities.

In the cytotoxic studies, lipids extracted from shellfish species showed varied levels of cytotoxicity against the studied cancer cells. The lipids extracted from the Argentine red shrimp showed the highest cytotoxicity against glioblastoma multiforme (T98G) cells, with an IC50 of $12.3 \mu \mathrm{g} / \mathrm{mL}$. In contrast, lipids of the long arm octopus were the most cytotoxic to lung carcinoma (AS49) cells, with an $\mathrm{IC}_{50}$ of $13.3 \mu \mathrm{g} / \mathrm{mL}$.

The composition of major lipophilic compounds and cytotoxicity data against several cancer types of cancer cells reported herein may be helpful in exploring the nutritional and anticancer potential of shellfish species.

The presence of a wide range of sterols (including isomeric forms) in shellfish species makes the qualitative analysis complicated. In the future, investigation with the aid of NMR could provide further information to confirm the identities. Moreover, sampling from several locations of the country can provide more comprehensive information on the contents of these nutritionally vital lipophilic constituents and their anticancer potential. 
Author Contributions: Conceptualization, J.S. and R.K.S.; methodology, J.S. and R.K.S.; validation, J.S., J.-W.O., Y.-S.K. and R.K.S.; formal analysis, J.S. and R.K.S.; investigation, J.S. and R.K.S.; resources, J.-W.O. and Y.-S.K.; data curation, J.S. and R.K.S.; writing-original draft preparation, J.S. and R.K.S.; writing-review and editing, J.S., E.-Y.K., J.-W.O., X.S., Y.-S.K. and R.K.S.; supervision, J.-W.O. and Y.-S.K.; project administration, M.-H.S. and J.-W.Y.; funding acquisition, R.K.S. and J.S. All authors have read and agreed to the published version of the manuscript.

Funding: This work was supported by grants (R.K.S.: 2019R1G1A1006815; J.S.: 2021R1I1A1A01057504; and J.-W.O.: 2017H1D3A1A01052995) of the National Research Foundation (NRF) funded by the Ministry of Science and ICT (MSIT), Korea. The article processing charges were supported by the grant 2021R1I1A1A01057504 of NRF, MSIT, Korea.

Institutional Review Board Statement: Not applicable.

Informed Consent Statement: Not applicable.

Data Availability Statement: Data is contained within the article.

Acknowledgments: This paper was supported by the KU research professor program of Konkuk University, Seoul, Korea.

Conflicts of Interest: The authors declare no conflict of interest.

Appendix A. Outline of the Method Used for Extraction of Crude Lipids from the Shellfish Species

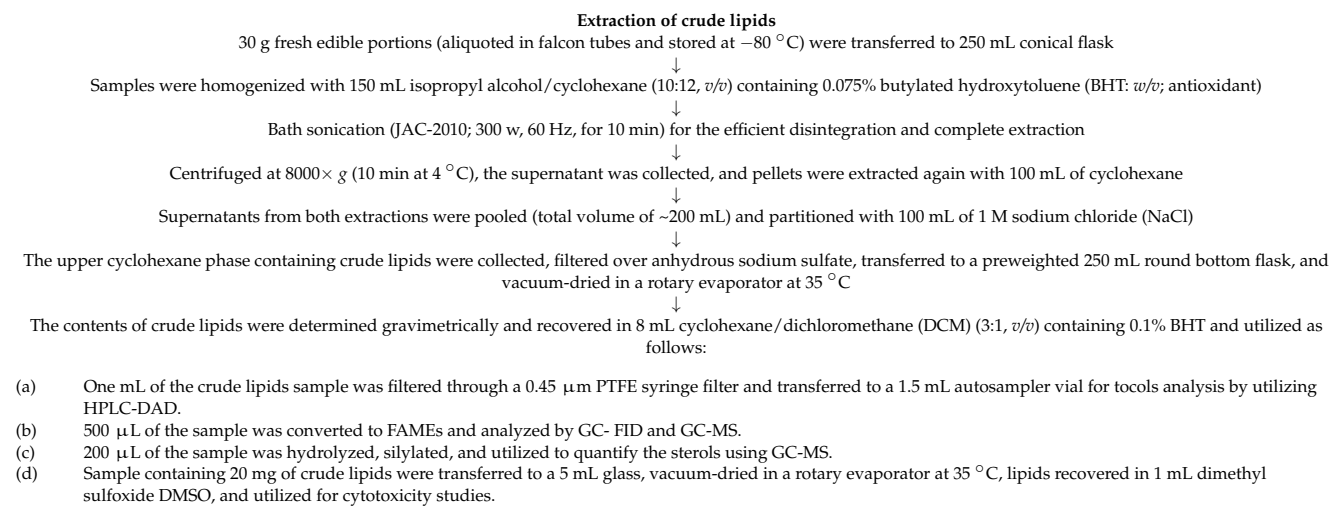

Appendix B. Outline of Methods Used for the Hydrolysis and Silylation of Sterols for GC-MS Analysis (A) and the Preparation of Fatty Acid Methyl Esters (B)

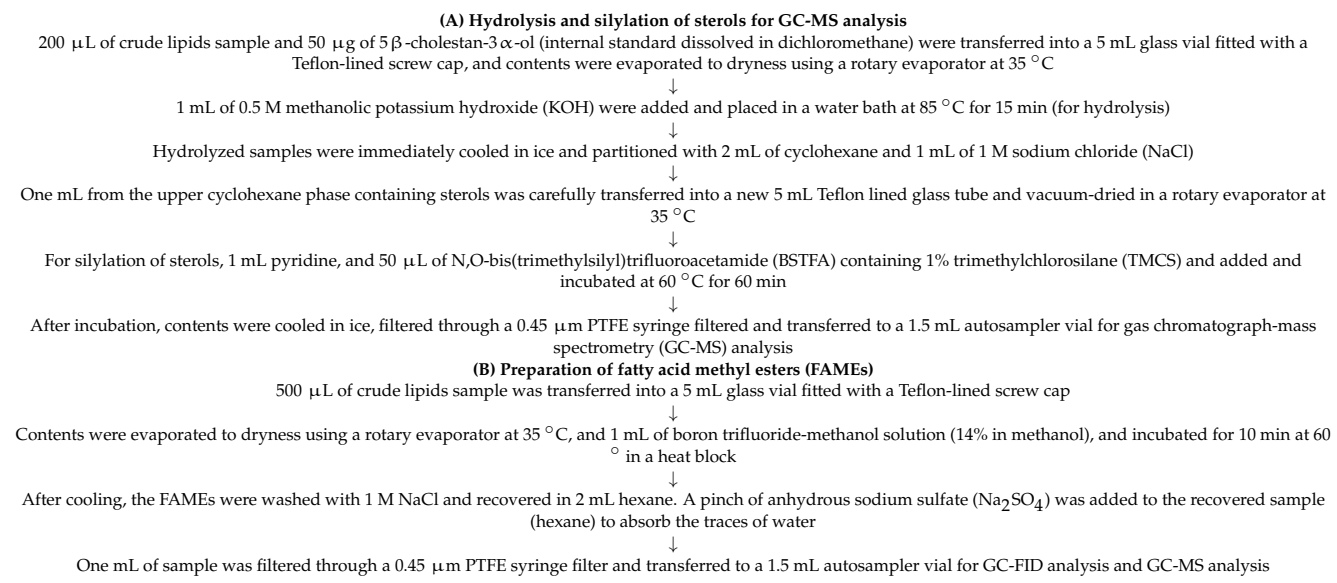


Appendix C. The Mass Spectrometric Fragmentation Pattern of Unknown Sterols Observed in the Present Investigation
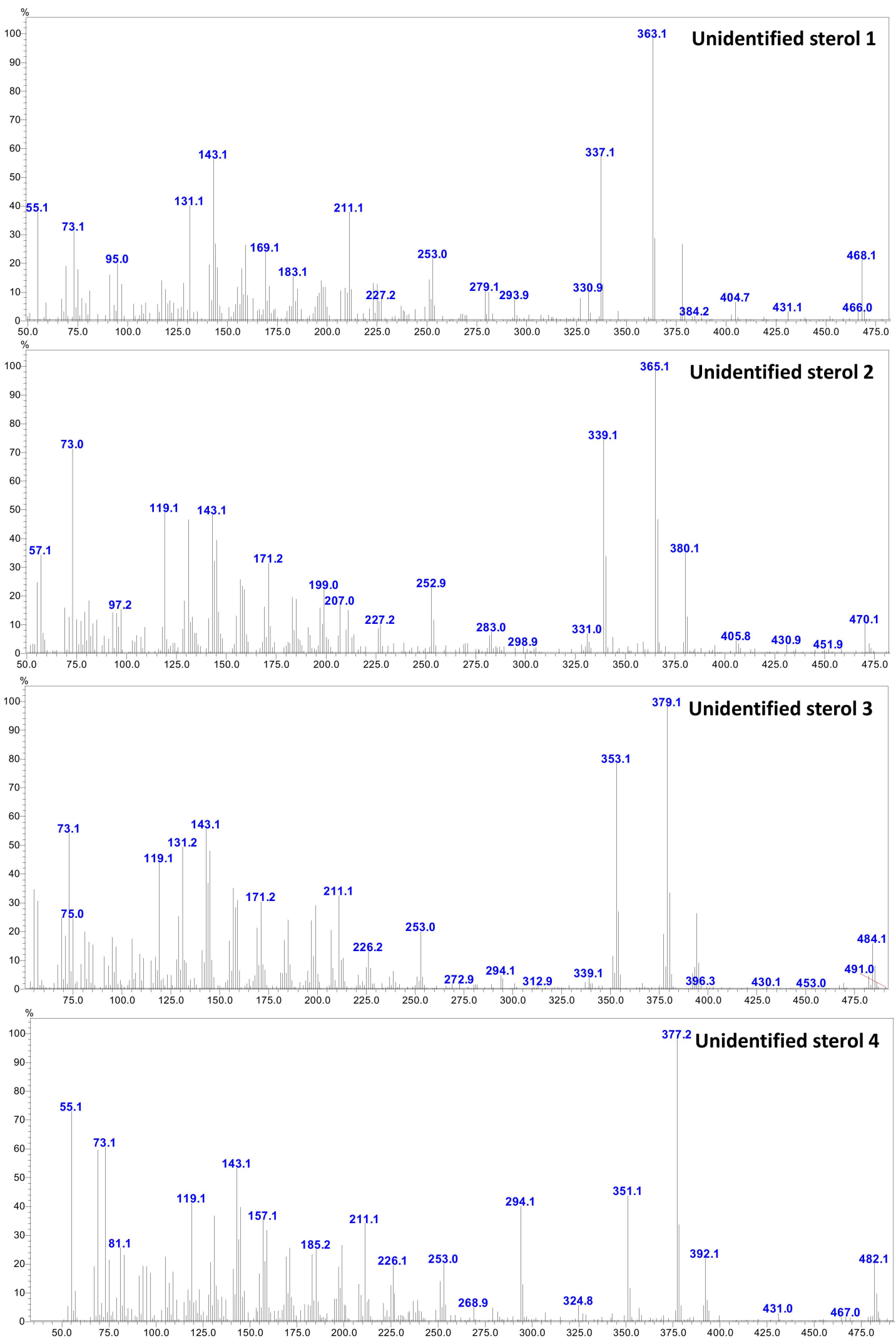

Figure A1. The mass spectrometric fragmentation pattern of unknown sterols observed in the present investigation. 


\section{References}

1. Ghasemi Fard, S.; Wang, F.; Sinclair, A.J.; Elliott, G.; Turchini, G.M. How Does High DHA Fish Oil Affect Health? A Systematic Review of Evidence. Crit. Rev. Food Sci. Nutr. 2019, 59, 1684-1727. [CrossRef] [PubMed]

2. Belayev, L.; Hong, S.H.; Menghani, H.; Marcell, S.J.; Obenaus, A.; Freitas, R.S.; Khoutorova, L.; Balaszczuk, V.; Jun, B.; Oriá, R.B.; et al. Docosanoids Promote Neurogenesis and Angiogenesis, Blood-Brain Barrier Integrity, Penumbra Protection, and Neurobehavioral Recovery after Experimental Ischemic Stroke. Mol. Neurobiol. 2018, 55, 7090-7106. [CrossRef] [PubMed]

3. Mayurasakorn, K.; Niatsetskaya, Z.V.; Sosunov, S.A.; Williams, J.J.; Zirpoli, H.; Vlasakov, I.; Deckelbaum, R.J.; Ten, V.S. DHA but Not EPA Emulsions Preserve Neurological and Mitochondrial Function after Brain Hypoxia-Ischemia in Neonatal Mice. PLoS ONE 2016, 11, e0160870. [CrossRef] [PubMed]

4. Phillips, K.; Ruggio, D.M.; Exler, J.; Patterson, K.Y. Sterol Composition of Shellfish Species Commonly Consumed in the United States. Food Nutr. Res. 2012, 56, 18931. [CrossRef] [PubMed]

5. Gylling, H.; Plat, J.; Turley, S.; Ginsberg, H.N.; Ellegard, L.; Jessup, W.; Jones, P.J.; Lutjohann, D.; Maerz, W.; Masana, L.; et al. Plant Sterols and Plant Stanols in the Management of Dyslipidaemia and Prevention of Cardiovascular Disease. Atherosclerosis 2014, 232, 346-360. [CrossRef]

6. Ras, R.T.; Geleijnse, J.M.; Trautwein, E.A. LDL-Cholesterol-Lowering Effect of Plant Sterols and Stanols Across Different Dose ranges: A Meta-Analysis of Randomised Controlled Studies. Br. J. Nutr. 2014, 112, 214-219. [CrossRef]

7. De Smet, E.; Mensink, R.P.; Plat, J. Effects of Plant Sterols and Stanols on Intestinal Cholesterol Metabolism: Suggested Mechanisms from Past to Present. Mol. Nutr. Food Res. 2012, 56, 1058-1072. [CrossRef]

8. Yoshida, Y.; Niki, E. Antioxidant Effects of Phytosterol and its Components. J. Nutr. Sci. Vitaminol. 2003, 49, 277-280. [CrossRef]

9. Awad, A.B.; Fink, C.S. Phytosterols as Anticancer Dietary Components: Evidence and Mechanism of Action. J. Nutr. 2000, 130, 2127-2130. [CrossRef] [PubMed]

10. Othman, R.A.; Moghadasian, M.H. Beyond Cholesterol-Lowering Effects of Plant Sterols: Clinical and Experimental Evidence of Anti-Inflammatory Properties. Nutr. Rev. 2011, 69, 371-382. [CrossRef]

11. Kuhnlein, H.V.; Barthet, V.; Farren, A.; Falahi, E.; Leggee, D.; Receveur, O.; Berti, P. Vitamins A, D, and E in Canadian Arctic traditional Food and Adult Diets. J. Food Compos. Anal. 2006, 19, 495-506. [CrossRef]

12. Ungurianu, A.; Zanfirescu, A.; Nițulescu, G.; Margină, D. Vitamin E beyond Its Antioxidant Label. Antioxidants 2021, 10, 634. [CrossRef]

13. Saini, R.K.; Keum, Y.S. Tocopherols and Tocotrienols in Plants and Their Products: A Review on Methods of Extraction, Chromatographic Separation, and Detection. Food Res. Int. 2016, 82, 59-70. [CrossRef]

14. Hikihara, R.; Yamasaki, Y.; Shikata, T.; Nakayama, N.; Sakamoto, S.; Kato, S.; Hatate, H.; Tanaka, R. Analysis of Phytosterol, Fatty Acid, and Carotenoid Composition of 19 Microalgae and 6 Bivalve Species. J. Aquat. Food Prod. Technol. 2020, 29, 461-479. [CrossRef]

15. Ozogul, F.; Kuley, E.; Ozogul, Y. Sterol Content of Fish, Crustacea and Mollusc: Effects of Cooking Methods. Int. J. Food Prop. 2015, 18, 2026-2041. [CrossRef]

16. Bragagnolo, N.; Rodriguez-Amaya, D.B. Total Lipid, Cholesterol, and Fatty Acids of Farmed Freshwater Prawn (Macrobrachium rosenbergii) and Wild Marine Shrimp (Penaeus brasiliensis, Penaeus schimitti, Xiphopenaeus kroyeri). J. Food Compos. Anal. 2001, 14, 359-369. [CrossRef]

17. Chen, L.; Wang, Q.; Cong, X.; Yu, F.; Xue, Y.; Tang, Q.; Xue, C.; Liu, Y.; Li, Z. Seasonal Variations in Total Lipid and Fatty Acid Composition of Haliotis discus Hannai from Three Different Production Zones. J. Aquat. Food Prod. Technol. 2021, 30, 1-10. [CrossRef]

18. Lu, T.; Shen, Y.; Cui, G.-X.; Yin, F.-W.; Yu, Z.-L.; Zhou, D.-Y. Detailed Analysis of Lipids in Edible Viscera and Muscles of Cooked Crabs Portunus trituberculatus and Portunus pelagicus. J. Aquat. Food Prod. Technol. 2020, 29, 391-406. [CrossRef]

19. Saito, H.; Aono, H. Characteristics of Lipid and Fatty acid of Marine Gastropod Turbo cornutus: High Levels of Arachidonic and n-3 Docosapentaenoic Acid. Food Chem. 2014, 145, 135-144. [CrossRef] [PubMed]

20. Fernández-Reiriz, M.J.; Labarta, U.; Albentosa, M.; Pérez-Camacho, A. Lipid Composition of Ruditapes philippinarum Spat: Effect of Ration and Diet Quality. Comp. Biochem. Physiol. Part B Biochem. Mol. Biol. 2006, 144, 229-237. [CrossRef] [PubMed]

21. Tsape, K.; Sinanoglou, V.J.; Miniadis-Meimaroglou, S. Comparative Analysis of the Fatty Acid and Sterol Profiles of Widely Consumed Mediterranean Crustacean Species. Food Chem. 2010, 122, 292-299. [CrossRef]

22. Saini, R.K.; Song, M.-H.; Rengasamy, K.R.R.; Ko, E.-Y.; Keum, Y.-S. Red Shrimp Are a Rich Source of Nutritionally Vital Lipophilic Compounds: A Comparative Study among Edible Flesh and Processing Waste. Foods 2020, 9, 1179. [CrossRef] [PubMed]

23. Cruz, R.; Casal, S.; Mendes, E.; Costa, A.; Santos, C.; Morais, S. Validation of a Single-Extraction Procedure for Sequential Analysis of Vitamin E, Cholesterol, Fatty Acids, and Total Fat in Seafood. Food Anal. Methods 2013, 6, 1196-1204. [CrossRef]

24. Saini, R.K.; Keum, Y.-S. Carotenoid Extraction Methods: A Review of Recent Developments. Food Chem. 2018, $240,90-103$. [CrossRef]

25. Biswas, S.K.; Kim, D.-E.; Keum, Y.-S.; Saini, R.K. Metabolite Profiling and Antioxidant Activities of White, Red, and Black Rice (Oryza sativa L.) Grains. J. Food Meas. Charact. 2018, 12, 2484-2492. [CrossRef]

26. Saini, R.K.; Rauf, A.; Khalil, A.A.; Ko, E.-Y.; Keum, Y.-S.; Anwar, S.; Alamri, A.; Rengasamy, K.R.R. Edible Mushrooms Show Significant Differences in Sterols and Fatty Acid Compositions. S. Afr. J. Bot. 2021, 141, 344-356. [CrossRef]

27. Ulbricht, T.L.V.; Southgate, D.A.T. Coronary Heart Disease: Seven Dietary Factors. Lancet 1991, 338, 985-992. [CrossRef] 
28. Wołoszyn, J.; Haraf, G.; Okruszek, A.; Wereńska, M.; Goluch, Z.; Teleszko, M. Fatty Acid Profiles and Health Lipid Indices in the Breast Muscles of Local Polish Goose Varieties. Poult. Sci. 2020, 99, 1216-1224. [CrossRef]

29. Chen, J.; Liu, H. Nutritional Indices for Assessing Fatty Acids: A Mini-Review. Int. J. Mol. Sci. 2020, 21, 5695. [CrossRef]

30. Santos-Silva, J.; Mendes, I.A.; Bessa, R.J.B. The Effect of Genotype, Feeding System and Slaughter Weight on the Quality of Light Lambs: 1. Growth, Carcass Composition and Meat Quality. Livest. Prod. Sci. 2002, 76, 17-25. [CrossRef]

31. Saini, R.K.; Song, M.-H.; Yu, J.-W.; Shang, X.; Keum, Y.-S. Phytosterol Profiling of Apiaceae Family Seeds Spices Using GC-MS. Foods 2021, 10, 2378. [CrossRef]

32. Assefa, A.D.; Keum, Y.S.; Saini, R.K. A Comprehensive Study of Polyphenols Contents and Antioxidant Potential of 39 Widely Used Spices and Food Condiments. J. Food Meas. Charact. 2018, 12, 1548-1555. [CrossRef]

33. Thaipong, K.; Boonprakob, U.; Crosby, K.; Cisneros-Zevallos, L.; Hawkins Byrne, D. Comparison of ABTS, DPPH, FRAP, and ORAC Assays for Estimating Antioxidant Activity from Guava Fruit Extracts. J. Food Compos. Anal. 2006, 19, 669-675. [CrossRef]

34. Goad, L.J.; Akihisa, T. (Eds.) Mass Spectrometry of Sterols. In Analysis of Sterols; Springer: Berlin/Heidelberg, Germany, 1997; pp. 152-196. [CrossRef]

35. Kanazawa, A. Sterols in Marine Invertebrates. Fish. Sci. 2001, 67, 997-1007. [CrossRef]

36. Zhu, H.; Chen, Z.-Y. Do We No Longer Need to Worry about Dietary Cholesterol? J. Agric. Food Chem. 2017, 65, 9931-9933. [CrossRef]

37. Samson, F.P.; Patrick, A.T.; Fabunmi, T.E.; Yahaya, M.F.; Madu, J.; He, W.; Sripathi, S.R.; Tyndall, J.; Raji, H.; Jee, D.; et al. Oleic Acid, Cholesterol, and Linoleic Acid as Angiogenesis Initiators. ACS Omega 2020, 5, 20575-20585. [CrossRef]

38. Krauss, R.M.; Eckel, R.H.; Howard, B.; Appel, L.J.; Daniels, S.R.; Deckelbaum, R.J.; Erdman, J.W.; Kris-Etherton, P.; Goldberg, I.J.; Kotchen, T.A.; et al. AHA Dietary Guidelines. Circulation 2000, 102, 2284-2299. [CrossRef] [PubMed]

39. Committee, D.G.A. Dietary Guidelines for Americans 2015-2020; Government Printing Office: Washington, DC, USA, 2015.

40. Fu, Y.; Li, G.; Zhang, X.; Xing, G.; Hu, X.; Yang, L.; Li, D. Lipid Extract from Hard-Shelled Mussel (Mytilus coruscus) Improves Clinical Conditions of Patients with Rheumatoid Arthritis: A Randomized Controlled Trial. Nutrients 2015, 7, 625-645. [CrossRef] [PubMed]

41. Gómez-Estaca, J.; Calvo, M.M.; Álvarez-Acero, I.; Montero, P.; Gómez-Guillén, M.C. Characterization and Storage Stability of Astaxanthin Esters, Fatty Acid Profile and $\alpha$-Tocopherol of Lipid Extract from Shrimp (L. vannamei) Waste with Potential Applications as Food Ingredient. Food Chem. 2017, 216, 37-44. [CrossRef] [PubMed]

42. Takeungwongtrakul, S.; Benjakul, S.; H-kittikun, A. Lipids from Cephalothorax and Hepatopancreas of Pacific white Shrimp (Litopenaeus vannamei): Compositions and Deterioration as Affected by Iced Storage. Food Chem. 2012, 134, 2066-2074. [CrossRef]

43. Sriket, P.; Benjakul, S.; Visessanguan, W.; Kijroongrojana, K. Comparative Studies on Chemical Composition and Thermal Properties of Black Tiger Shrimp (Penaeus monodon) and White Shrimp (Penaeus vannamei) Meats. Food Chem. 2007, 103, 1199-1207. [CrossRef]

44. Drouin, G.; Rioux, V.; Legrand, P. The n-3 Docosapentaenoic Acid (DPA): A New Player in the n-3 Long Chain Polyunsaturated Fatty Acid Family. Biochimie 2019, 159, 36-48. [CrossRef] [PubMed]

45. Simopoulos, A.P. Evolutionary Aspects of the Dietary Omega-6/Omega-3 Fatty Acid Ratio: Medical Implications. In Evolutionary Thinking in Medicine; Springer: Berlin/Heidelberg, Germany, 2016; pp. 119-134.

46. Saini, R.K.; Keum, Y.S. Omega-3 and Omega-6 Polyunsaturated Fatty Acids: Dietary Sources, Metabolism, and Significance-A Review. Life Sci. 2018, 203, 255-267. [CrossRef] [PubMed]

47. Apak, R.; Özyürek, M.; Güçlü, K.; Çapanoğlu, E. Antioxidant Activity/Capacity Measurement. 2. Hydrogen Atom Transfer (HAT)-Based, Mixed-Mode (Electron Transfer (ET)/HAT), and Lipid Peroxidation Assays. J. Agric. Food Chem. 2016, 64, 1028-1045. [CrossRef]

48. Abraham, A.; Kattoor, A.J.; Saldeen, T.; Mehta, J.L. Vitamin E and its Anticancer Effects. Crit. Rev. Food Sci. Nutr. 2019, 59, 2831-2838. [CrossRef] [PubMed]

49. Brown, I.; Lee, J.; Sneddon, A.A.; Cascio, M.G.; Pertwee, R.G.; Wahle, K.W.J.; Rotondo, D.; Heys, S.D. Anticancer Effects of n-3 EPA and DHA and Their Endocannabinoid Derivatives on Breast Cancer Cell Growth and Invasion. Prostaglandins Leukot. Essent. Fat. Acids 2020, 156, 102024. [CrossRef]

50. Xu, Y.; Ryu, S.; Lee, Y.-K.; Lee, H.-J. Brassicasterol from Edible Aquacultural Hippocampus abdominalis Exerts an Anti-Cancer Effect by Dual-Targeting AKT and AR Signaling in Prostate Cancer. Biomedicines 2020, 8, 370. [CrossRef] 